\title{
Tropical Cyclogenesis Associated with Rossby Wave Energy Dispersion of a Preexisting Typhoon. Part II: Numerical Simulations*
}

\author{
Tim Li, Xuyang Ge, And Bin Wang \\ Department of Meteorology, and International Pacific Research Center, University of Hawaii at Manoa, Honolulu, Hawaii \\ YoNGTI ZHU \\ Shanghai Typhoon Institute, Shanghai, China
}

(Manuscript received 29 October 2004, in final form 7 June 2005)

\begin{abstract}
The cyclogenesis events associated with the tropical cyclone (TC) energy dispersion are simulated in a 3D model. A new TC with realistic dynamic and thermodynamic structures forms in the wake of a preexisting $\mathrm{TC}$ when a large-scale monsoon gyre or a monsoon shear line flow is present. Maximum vorticity generation appears in the planetary boundary layer (PBL) and the vorticity growth exhibits an oscillatory development. This oscillatory growth is also seen in the observed rainfall and cloud-top temperature fields. The diagnosis of the model output shows that the oscillatory development is attributed to the discharge and recharge of the PBL moisture and its interaction with convection and circulation. The moisture-convection feedback regulates the TC development through controlling the atmospheric stratification, raindrop-induced evaporative cooling and downdraft, PBL divergence, and vorticity generation. On one hand, ascending motion associated with deep convection transports moisture upward and leads to the discharge of PBL moisture and a convectively stable stratification. On the other hand, the convection-induced raindrops evaporate, leading to midlevel cooling and downdraft. The downdraft further leads to dryness and a reduction of equivalent potential temperature. This reduction along with the recharge of PBL moisture due to surface evaporation leads to reestablishment of a convectively unstable stratification and thus new convection.

Sensitivity experiments with both a single mesh (with a $15-\mathrm{km}$ resolution) and a nested mesh (with a 5-km resolution in the inner mesh) indicate that $\mathrm{TC}$ energy dispersion alone in a resting environment does not lead to cyclogenesis, suggesting the important role of the wave train-mean flow interaction. A proper initial condition for background wind and moisture fields is crucial for maintaining a continuous vorticity growth through the multioscillatory phases.
\end{abstract}

\section{Introduction}

Tropical cyclone (TC) genesis is the least understood phase of the TC life cycle. In Li and Fu (2006, hereafter Part I), a cyclogenesis scenario associated with the Rossby wave energy dispersion of a preexisting typhoon was revealed from Quick Scatterometer (QuikSCAT) and Tropical Rainfall Measuring Mission (TRMM) Microwave Imager (TMI) satellite data. In Part II, we

* SOEST Contribution Number 6743 and IPRC Contribution Number 355.

Corresponding author address: Prof. Tim Li, IPRC, and Dept. of Meteorology, University of Hawaii at Manoa, 2525 Correa Rd., Honolulu, HI 96822.

E-mail: timli@hawaii.edu simulate this cyclogenesis process in a 3D primitive equation model to investigate the specific physical processes that give rise to the cyclogenesis.

Most previous studies on TC energy dispersion (TCED) were confined to a barotropic framework with the focus on TC motion. For instance, using barotropic models, Chan and Williams (1987) and Fiorino and Elsberry (1989) showed that a cyclonic vortex experiences Rossby wave energy dispersion, resulting in an asymmetric structure with a cyclonic gyre to the west and an anticyclonic gyre to the east of the vortex center and the ventilation flow between the gyres that advects the vortex poleward and westward. Except for the isolated vortex that has a net zero angular momentum, most vortices are subject to energy dispersion due to Rossby wave radiation (Flierl 1984; Shapiro and Ooyama 1990; Luo 1994; Carr and Elsberry 1995). Luo (1994) studied 
the effect of Rossby energy dispersion on the TC motion and structure in a nondivergent barotropic model and showed that a wavelike wake formed as a result of Rossby energy dispersion from the vortex. Because of the leaking of energy, the vortex weakened with time in the model.

Holland (1995) made the first attempt to simulate the TCED-induced cyclogenesis in a 3D model in a resting environment. The convective heating in the model was proportional to low-level vorticity. Initializing the model with a cyclonic vortex on a $\beta$ plane in a quiescent environment, a wake with alternating anticyclonic and cyclonic vorticity developed because of the TCED process. Because of a positive feedback between the convective heating and the low-level vorticity, a new TC was generated in the wake with cyclonic circulation. The numerical experiment thus suggested that the TCED alone, without interactions with the mean flow, might lead to cyclogenesis.

As shown in Part I, not all TCED-induced Rossby wave trains finally lead to cyclogenesis. Favorable background (dynamic and thermodynamic) conditions may set the preferred timing and location for cyclogenesis, while unfavorable environmental conditions may prohibit TC formation. Our observational results motivate us to revisit this TCED-induced cyclogenesis problem using a 3D TC model with more realistic moist physical processes. In particular, we intend to investigate whether the wave train alone can lead to cyclogenesis or if a favorable background flow condition is required for such events. In addition to simulating the TCED-induced cyclogenesis in a 3D model, we will diagnose the model output to examine the dynamics and specific physical processes that give rise to the cyclogenesis, with a particular emphasis on feedbacks among the circulation, convection, and atmospheric moisture fields.

The rest of the paper is organized as follows. In section 2, we briefly introduce the model and the experimental design. The evolution of vorticity, humidity, vertical motion, and surface pressure associated with the TCED-induced cyclogenesis is presented in section 3 , and the role of atmospheric moisture in regulating convective circulation and stratification is described in section 4. Sensitivity of the model solution to initial moisture profiles and the model resolution is examined in section 5. Finally, a summary is given in the last section.

\section{The model and initial conditions}

The atmospheric model used in this study is the uniform grid version of the triply nested movable mesh primitive equation model (TCM3). A detailed descrip- tion of the model can be found in Wang (1999, 2001). The model is a hydrostatic primitive equation model formulated in Cartesian coordinates in the horizontal on either an $f$ or beta plane, with $\sigma$ (pressure normalized by the surface pressure) as the vertical coordinate. The model consists of 20 layers in the vertical from $\sigma=$ 0 to $\sigma=1$ with a substantial concentration of resolution within the planetary boundary layer (PBL). The model physics include an $E-\varepsilon$ turbulence closure scheme for subgrid scale vertical mixing above the surface layer (Detering and Etling 1985; Langland and Liou 1996), a modified Monin-Obukhov scheme for the surface flux calculations (Fairall et al. 1996), an explicit treatment of mixed ice phase cloud microphysics (Rutledge and Hobbs 1984; Reisner et al. 1998; Wang 1999, 2001), and a fourth-order horizontal diffusion with a deformationdependent diffusion coefficient. The model prognostic variables consist of zonal and meridional winds, surface pressure, temperature, turbulence kinetic energy and its dissipation rate, and mixing ratios of water vapor, cloud water, rainwater, cloud ice, snow, and graupel. This model has been used for the studies of various aspects of tropical cyclones (e.g., Wang 2002).

Although TCM3 provides movable mesh and triply nested function, we use a single mesh with a uniform grid spacing of $15 \mathrm{~km}$ in this study to reduce the potential mesh interface effect on the structure of the wave train due to Rossby wave energy dispersion. At this resolution, TCM3 uses both the explicit heating scheme for grid-resolving processes and the Tiedtke (1989) mass flux convective parameterization scheme to include the effect of subgrid-scale cumulus convection. The model domain covers $4500 \mathrm{~km}$ by $4500 \mathrm{~km}$ on a beta plane centered at $18^{\circ} \mathrm{N}$. A strong damping is specified at a sponge layer near the model lateral boundaries to prevent wave reflection into the interior. To test the sensitivity of the model simulation to the domain size, a sensitivity experiment with a larger domain $(6000 \mathrm{~km}$ by $6000 \mathrm{~km}$ ) is conducted, and the result shows that the model solution is not sensitive to the domain size.

Since the main focus of this study is to examine whether tropical cyclogenesis can occur in the wave train of a preexisting TC due to the Rossby wave energy dispersion and the possible role of the large-scale environmental flow in such a scenario, we thus first spin up a TC on a beta plane for 10 days such that a 3D Rossby wave train pattern is well developed. The initial condition used in the spinup is a symmetric vortex, which has a maximum tangential wind of $30 \mathrm{~m} \mathrm{~s}^{-1}$ at a radius of $100 \mathrm{~km}$ in a resting environment. The tangential wind has maximum amplitude at the surface and decreases gradually to 0 at about $100 \mathrm{hPa}$. The environmental temperature and moisture profiles are the 

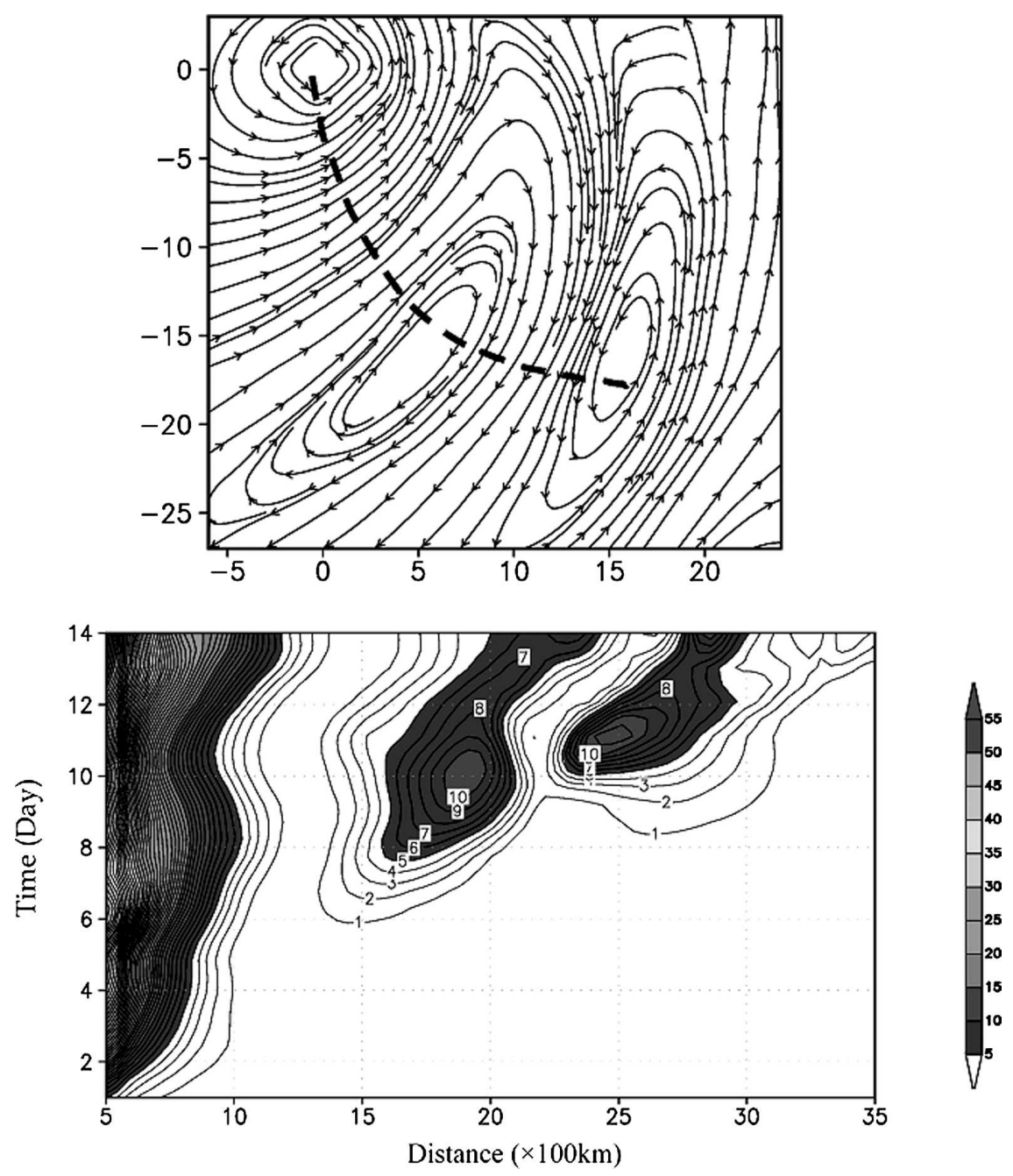

FIG. 1. (top) Streamline pattern of a Rossby wave train induced by TCED at $850 \mathrm{hPa}$ at day 10 and (bottom) the time evolution of $850-\mathrm{hPa}$ kinetic energy $\left(\mathrm{m}^{2} \mathrm{~s}^{-2}\right)$ along the southeastward direction (the dashed line in the top panel) from the TC center. Horizontal distance has a unit of $100 \mathrm{~km}$, relative to the TC center.

same as those used in Wang (2001) and represent the mean summertime conditions over the western Pacific.

Figure 1 illustrates the derived Rossby wave train pattern at day 10 and the time evolution of TC kinetic energy along the southeastward direction. The wave train is located in the southeast quadrant, with alternating cyclonic and anticyclonic circulation. The maximum kinetic energy propagates southeastward, radiating away from the TC center. The zonal wavelength of the wave train is on the order of $2000 \mathrm{~km}$. The simulated wave train patterns bear many similarities with those derived from the QuikSCAT observations as shown in Part I.

To study the mean flow effect, an idealized monsoon gyre (MG) pattern is specified (see the upper panel of Fig. 2). This MG has a size of $3000 \mathrm{~km}$ and a central minimum pressure $2-3 \mathrm{hPa}$ lower than the surrounding environment. The maximum wind of the $\mathrm{MG}$ is about 5 $\mathrm{m} \mathrm{s}^{-1}$ at a radius of $300 \mathrm{~km}$ from the center. The relative humidity (RH) near the MG center is set to be a few percentages (about $6 \%$ at the surface and 3\% at $500 \mathrm{hPa}$ ) higher than the surrounding to mimic Ekmanpumping-induced boundary layer moisture convergence. Both the pattern and strength of the MG are close to the observed composites (Ritchie and Holland 1999; see the lower panel of Fig. 2). The idealized MG has a baroclinic structure with cyclonic circulation below $500 \mathrm{hPa}$ and anticyclonic circulation above (not shown).

Of particular interest is the appearance of mesoscale eddies/vortices in the cyclonic vorticity region of the wave train. These eddies are generated possibly be- 



FIG. 2. (top) Idealized and (bottom) observed monsoon gyre patterns: (a) surface pressure field (hPa), (b) wind $\left(\mathrm{m} \mathrm{s}^{-1}\right)$ and vorticity $\left(10^{-5} \mathrm{~s}^{-1}\right)$ fields at $850 \mathrm{hPa},(\mathrm{c})$ surface pressure $(\mathrm{hPa})$, and (d) $850-\mathrm{hPa}$ streamline. Horizontal distance in (a) and (b) has a unit of $100 \mathrm{~km}$, relative to the monsoon gyre center. The observed composite is from Ritchie and Holland (1999).

cause of processes associated with the TC inner-core dynamics and TC energy dispersion, and they are clearly present at end of the 10-day integration with an idealized initial symmetric TC and with no mean flow. The mesoscale vortices may exert important influences on TC formation via either vortex merging (Simpson et al. 1997; Hendricks et al. 2004) or axisymmetrization (Möller and Montgomery 1999, 2000). Superposition of the Rossby wave train on the background monsoon gyre flow shows a much organized circulation structure (Fig. 3). Eight numerical experiments are designed (see Table 1) to investigate the various aspects of tropical cyclogenesis associated with the TC Rossby wave energy dispersion.

\section{TCED-induced cyclogenesis}

The following three experiments are first conducted to understand the role of the mean flow. In the first experiment (Exp1), the model is initialized with the
Rossby wave train only. This experiment is designed to examine whether the wave train alone can develop into a TC in a resting environment. In the second experiment (Exp2), we examine how the background $\mathrm{MG}$ evolves with time without the involvement of the TCED-induced Rossby wave train. In the third experiment (Exp3), the Rossby wave train is superposed on the MG as the initial condition. This experiment is thus designed to examine whether the wave train-MG interaction may lead to cyclogenesis.

Figure 4 shows the evolution of the minimum sea level pressure (MSLP) in the three experiments. While the wave train alone or the MG alone does not cause cyclogenesis, the superposition of wave train and $\mathrm{MG}$ leads to the generation of a new TC. At day 6, the MSLP drops down to $975 \mathrm{hPa}$. This result suggests that a proper mean flow pattern is essential for the TCEDinduced wave train to develop into a TC. The new TC development in this experiment is consistent with Emanuel's finite-amplitude nature of TC genesis 


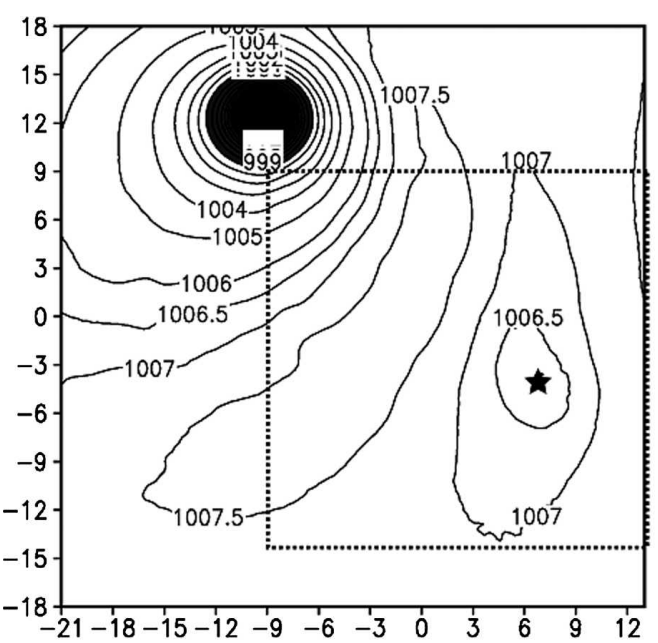

$(\times 100 \mathrm{~km})$

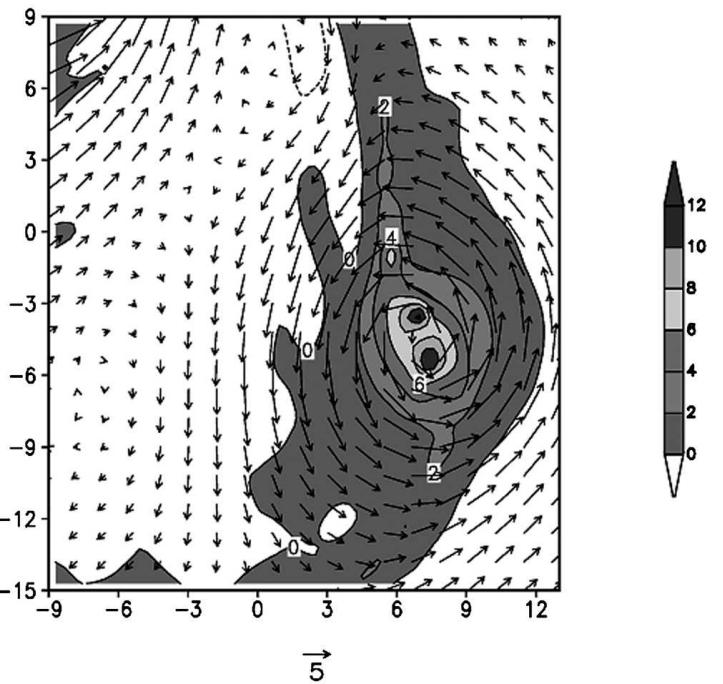

FIG. 3. (left) Surface pressure pattern (hPa) of the TCED-induced wave train and (right) the fine structure of vorticity perturbations at $850 \mathrm{hPa}\left(10^{-5} \mathrm{~s}^{-1}\right)$ in the cyclonic circulation region of the wave train (dashed square region in the left panel). Superposed in the right panel is the sum of the wave train and monsoon gyre surface wind vectors $\left(\mathrm{m} \mathrm{s}^{-1}\right)$. A star " $\star$ " denotes the superposed monsoon gyre center location. Horizontal distance has a unit of $100 \mathrm{~km}$, and $(0,0)$ denotes the center of the model domain.

(Emanuel 1989). As demonstrated by Emanuel, to allow the wind-induced surface heat exchange (WISHE) mechanism to operate effectively, the initial vortex should have an initial finite-amplitude in the surface winds. In either the wave train or the MG experiment, the maximum surface winds are only about $5-6 \mathrm{~m} \mathrm{~s}^{-1}$, which is not strong enough to spin up the vortex through the WISHE mechanism. In the third experiment, the surface winds are almost doubled and thus the WISHE effectively spins up the vortex, leading to the TC genesis. The lower panels of Fig. 4 illustrate the evolution of the surface pressure fields in the third experiment. A low pressure system develops slowly in the wake of a preexisting TC from day 1 to day 3 . It then deepens much faster and reaches $980 \mathrm{hPa}$ at day 5, even though at that time the previous TC has weakened.

The simulated new vortex exhibits realistic TC characteristics (Fig. 5), including a tilted eyewall, a vigorous in-up-and-out mean secondary circulation with mean subsidence in the eye region. The maximum tangential wind is about $35 \mathrm{~m} \mathrm{~s}^{-1}$ at a radius of about $60 \mathrm{~km}$ (Fig. $5 a)$. Strong inflow occurs in the boundary layer with maximum inflow of about $6 \mathrm{~m} \mathrm{~s}^{-1}$ just outside the radius of maximum tangential wind. A broad outflow layer is located in the upper troposphere outside the eyewall (Fig. 5b). The simulated cyclone has a warm core structure with a maximum temperature anomaly of $6 \mathrm{~K}$ around $200-300 \mathrm{hPa}$ (Fig. 5d). There is weak descending motion in the eye and a strong updraft near the eyewall, the latter tilting outward with height (Fig.
$5 \mathrm{c})$. Note also that there is a downdraft near the outer edge of the eyewall in the middle levels, which produces a cold and dry pool with relatively low equivalent potential temperature in the lower troposphere (not shown).

Figure 6 shows the evolution of vorticity, relative humidity, vertical velocity and rainfall rate averaged over a $120 \mathrm{~km}$ by $120 \mathrm{~km}$ domain centered at the maximum low-level vorticity. Two important features in the vorticity field are worth noting. First, maximum vorticity generation occurs in the PBL, and with the TC development, the cyclonic vorticity gradually penetrates

TABLE 1. List of major numerical experiments.

\begin{tabular}{|c|c|}
\hline $\begin{array}{l}\text { Experiment } \\
\text { symbol }\end{array}$ & Description \\
\hline Exp1 & $\begin{array}{l}\text { The Rossby wave train only in a resting } \\
\text { environment }\end{array}$ \\
\hline Exp2 & The monsoon gyre only (no Rossby wave train) \\
\hline Exp3 & The Rossby wave train plus the monsoon gyre \\
\hline Exp4 & $\begin{array}{l}\text { Same as Exp3 except with a reduced initial } \\
\text { background RH profile near the monsoon gyre } \\
\text { center }\end{array}$ \\
\hline Exp5 & $\begin{array}{l}\text { Same as Exp2 except with a reduced initial } \mathrm{RH} \\
\text { profile near the monsoon gyre center }\end{array}$ \\
\hline Exp6 & $\begin{array}{l}\text { Same as Exp1 except with a nested grid model } \\
\text { with the finest resolution of } 5 \mathrm{~km}\end{array}$ \\
\hline Exp7 & $\begin{array}{l}\text { Same as Exp3 except with the monsoon shear line } \\
\text { background flow pattern }\end{array}$ \\
\hline Exp8 & $\begin{array}{l}\text { Same as Exp3 except no monsoon gyre flow } \\
\text { (but keep the monsoon gyre moisture) }\end{array}$ \\
\hline
\end{tabular}



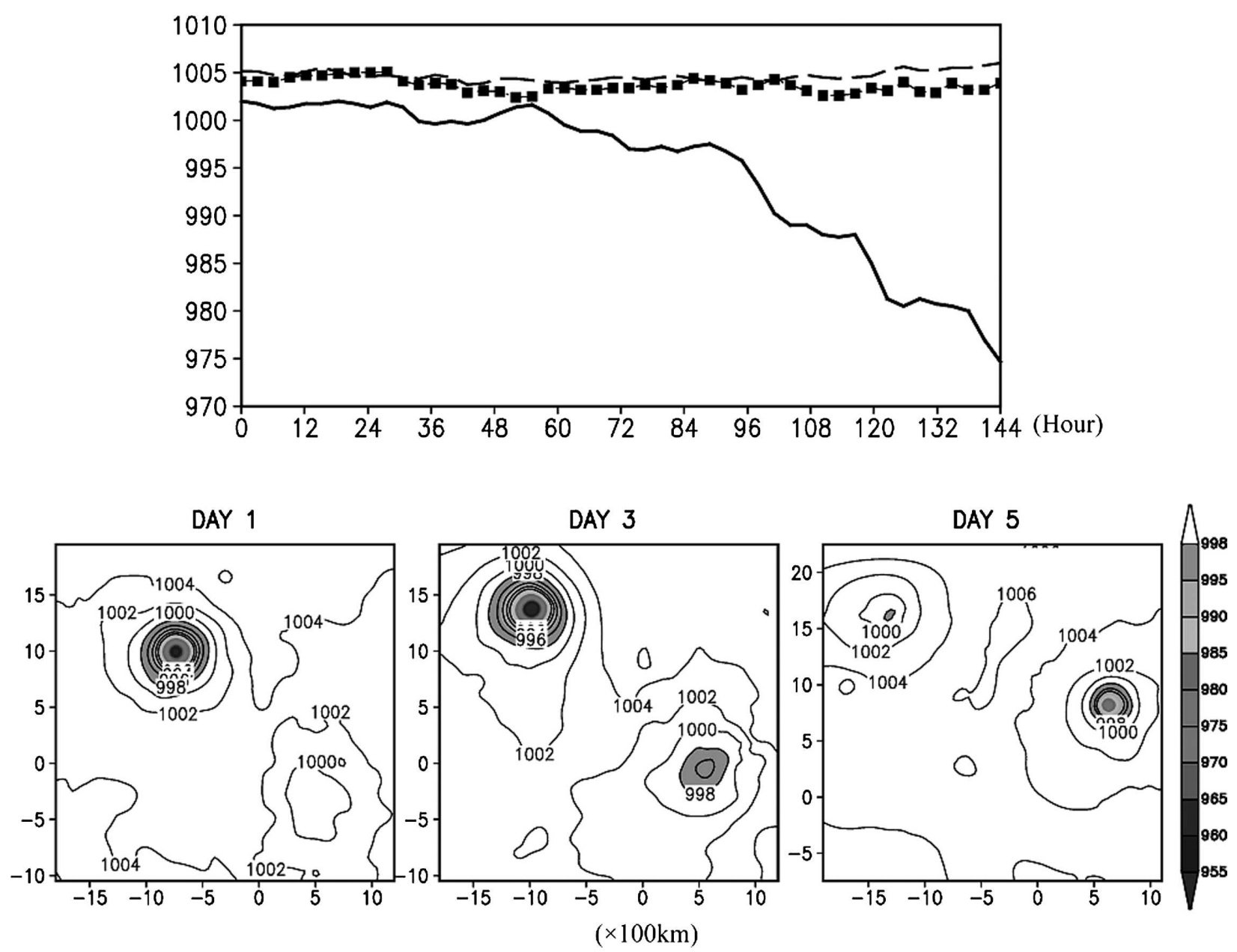

FIG. 4. (top) Time evolution of minimum sea level pressure fields for Exp1 (dashed line), Exp2 (dotted line), and Exp3 (solid line). (bottom) The surface pressure (hPa) patterns at days 1, 3, and 5 in Exp3.

from the PBL into the middle and upper troposphere. Secondly, during the initial development, the PBL vorticity experiences an oscillatory growth. For instance, the vorticity grows rapidly between hours 60 and 64, slows down or even decays afterwards, and reintensifies at hours $72-78$. The averaged rain rate also exhibits a clear oscillatory evolution with periods ranged from 9 to $15 \mathrm{~h}$. This oscillation feature is still clearly present in the vorticity or rainfall field averaged over a larger domain (e.g., $240 \mathrm{~km}$ by $240 \mathrm{~km}$ ).

The oscillatory vorticity development is closely related to the evolution of vertical motion and relative humidity fields. It is seen from Fig. 6 that there are alternations of updraft and downdraft, corresponding well to convectively active and break phases. While the ascending motion associated with penetrated convection leads to PBL convergence and thus a positive vorticity tendency, the downdraft leads to PBL divergence and thus a negative vorticity tendency. A vorticity bud- get analysis indicates that the generation of the PBL vorticity in the model is primarily attributed to the divergence term. The vertical motion regulates the moisture profile through vertical moisture transport, leading to the alternative change of relative humidity in midtroposphere.

\section{Effect of discharge/recharge of PBL moisture in TC oscillatory development}

A question related to the vortex development is why the vorticity experiences an oscillatory growth. The cause of the oscillatory vorticity growth might be attributed to the change in PBL divergence and vertical motion, but what causes the alternation of convectively active and break phases? To understand the interactive nature of the convection, moisture and circulation and the fundamental cause of the oscillatory vorticity growth, a composite of five (vorticity) intensifying and 


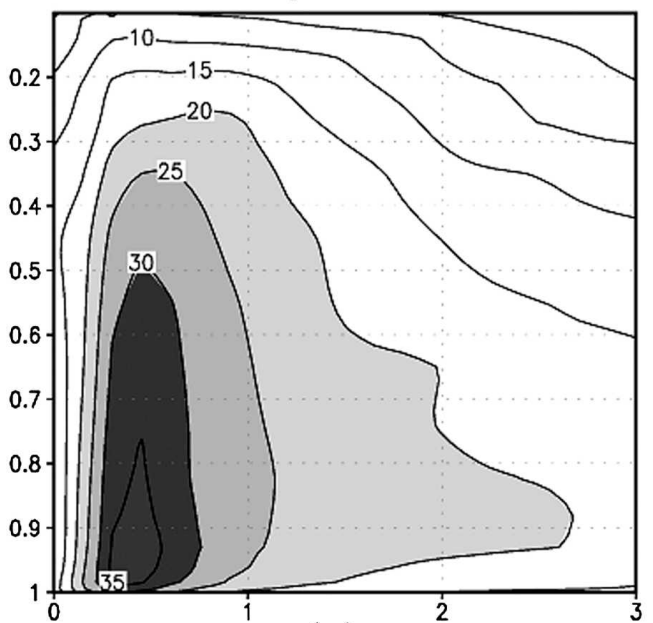

(a)

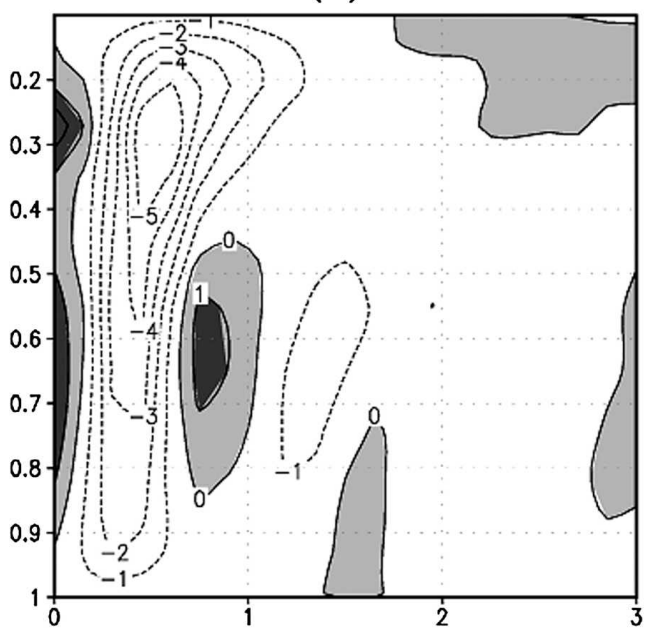

(c)

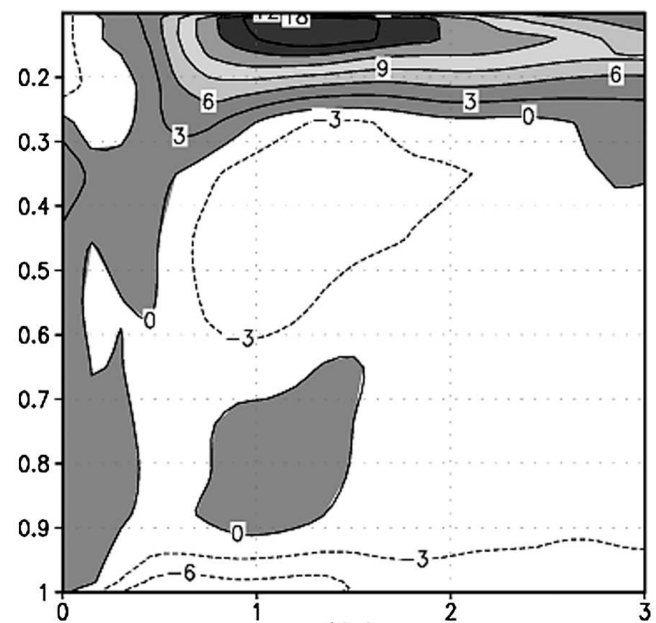

(b)

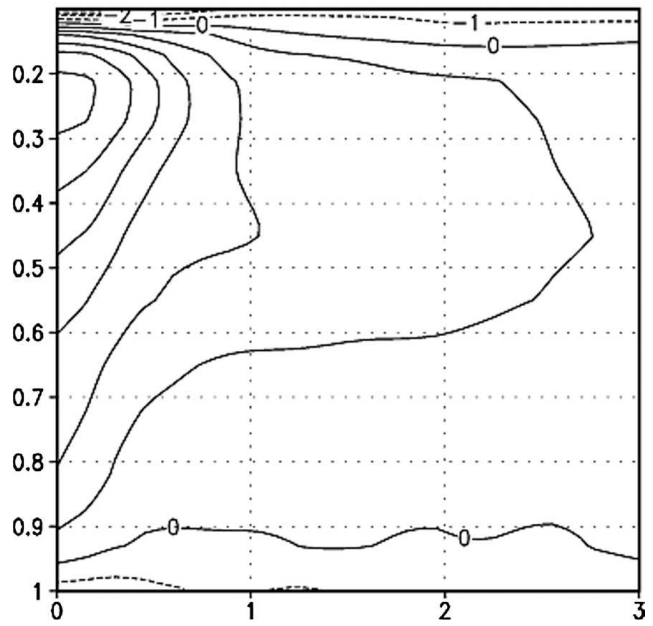

(d)

FIG. 5. The vertical-radial section of (a) tangential wind $\left(\mathrm{m} \mathrm{s}^{-1}\right)$, (b) radial wind $\left(\mathrm{m} \mathrm{s}^{-1}\right)$, (c) vertical velocity in the sigma coordinate $\left(10^{-5} \mathrm{~s}^{-1}\right)$, and (d) temperature anomaly (contour interval is $1 \mathrm{~K}$ ) at day 6 in Exp3. The horizontal axis is radius and the vertical axis is the sigma level.

weakening phases during the initial $(12 \sim 72 \mathrm{~h})$ period was constructed (see Fig. 7). Here $-180^{\circ}$ and $180^{\circ}$ represent the intensifying (or convectively active) phases characterized by maximum ascending motion throughout the troposphere. Between $-90^{\circ}$ and $0^{\circ}$ is the weakening (or convectively break) phase characterized by downdraft and low-level divergence. The sequence of physical mechanisms that can account for such an oscillatory growth is hypothesized as follows. Surface heating and the accumulation of PBL moisture lead to convectively unstable stratification and thus convection. Convective heating and upward motion induce low-level convergence, leading to increase of PBL vorticity. The upward transport of water vapor, on one hand, discharges the PBL moisture, and, on the other hand, reduces convective instability and stabilizes the atmospheric stratification. Meanwhile, the evaporation of precipitable water or raindrops leads to diabatic cooling (i.e., a negative latent heat rate, see Fig. 7e) and thus downdraft in the middle-to-lower troposphere (Fig. 7f). This leads to low-level divergence and a decrease in PBL vorticity (Fig. 7a,b). As the downdraft dries the middle-to-lower troposphere and reduces equivalent potential temperature, latent heat fluxes and evaporation from ocean surface recharge the PBL moisture. This leads to the rebuilding-up of convectively unstable stratification, and a new cycle begins.

The composite results above support the notion that the moisture and convective circulation feedback is crucial in leading to the oscillatory vorticity development. 

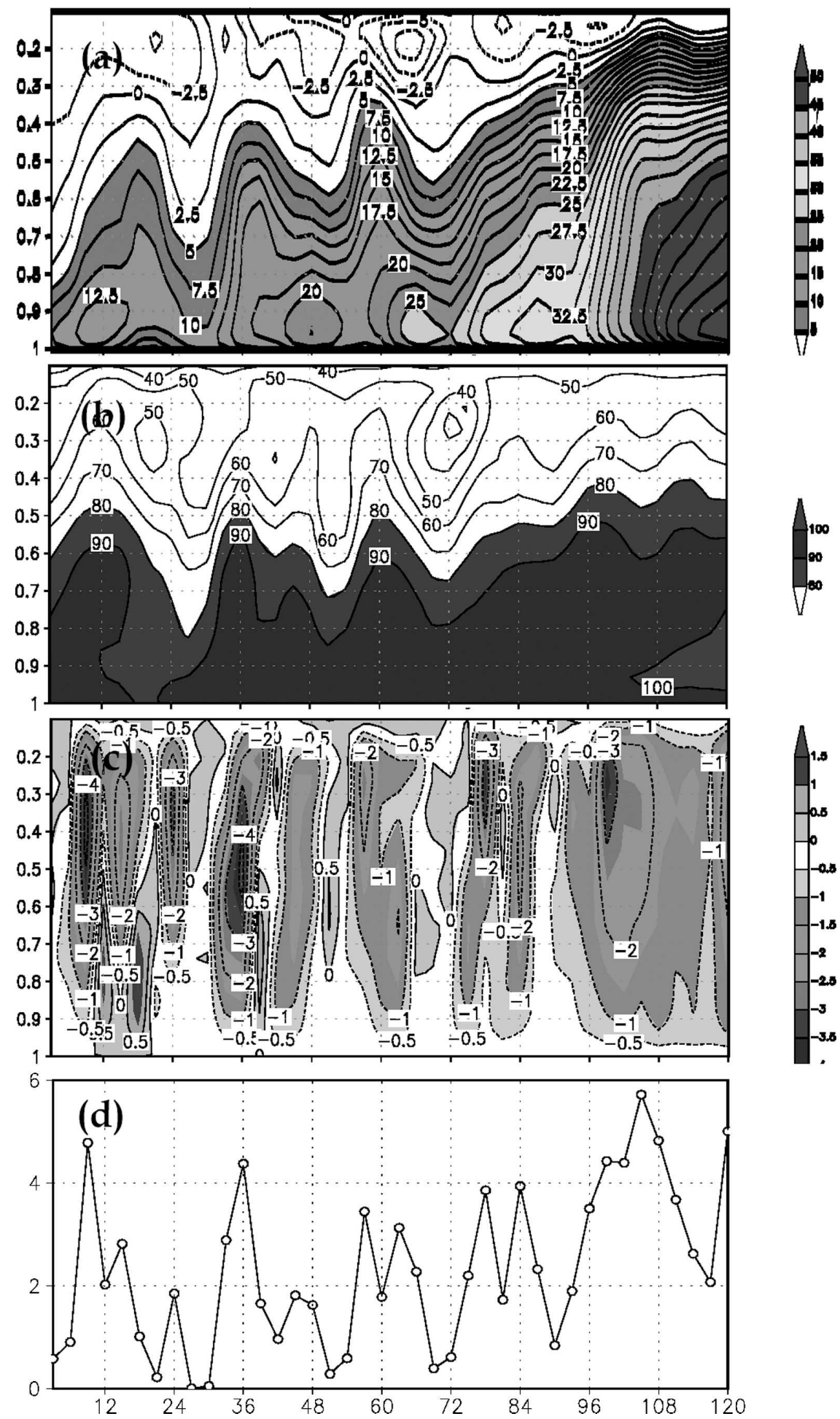

FIG. 6. Time evolution of (a) vertical profile of vorticity $\left(10^{-5} \mathrm{~s}^{-1}\right)$, (b) relative humidity $(\%)$, (c) vertical motion $\left(10^{-5} \mathrm{~s}^{-1}\right)$, and (d) rainfall rate $\left(\mathrm{mm} \mathrm{h}^{-1}\right)$ averaged over a $120 \mathrm{~km} \times$ $120 \mathrm{~km}$ domain centered at the low-level maximum vorticity in Exp3. The horizontal axis is time in hours and the vertical axis in (a)-(c) is the sigma level. 


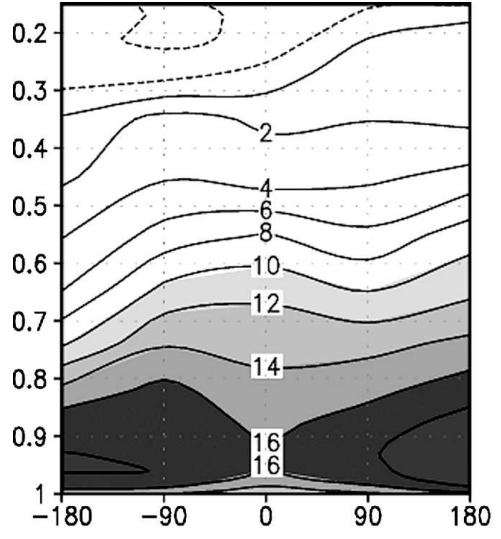

(a)

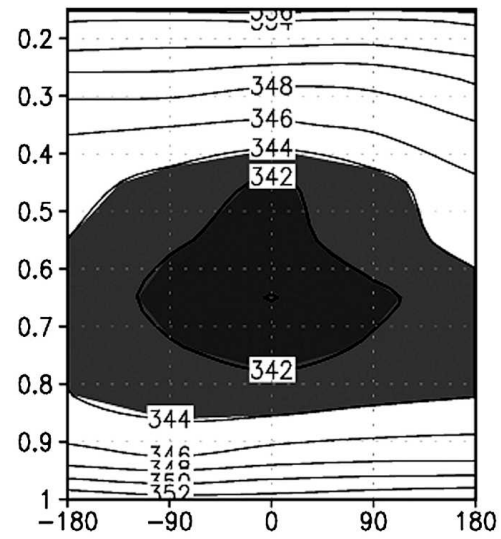

(d)

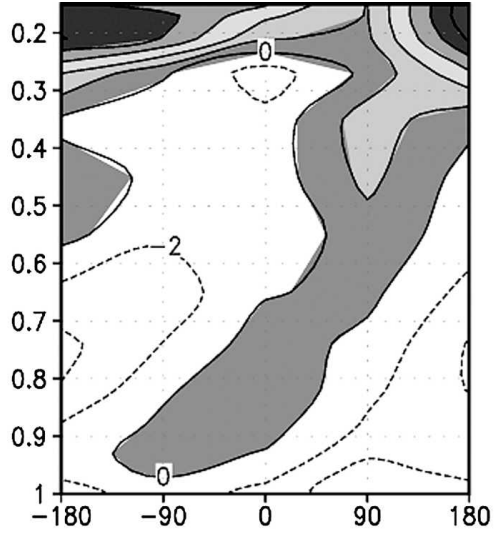

(b)

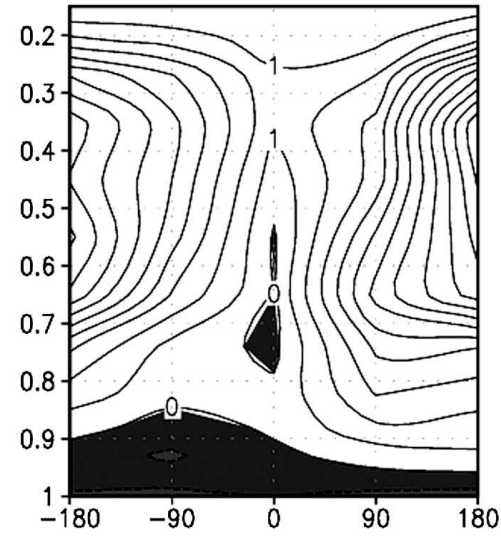

(e)

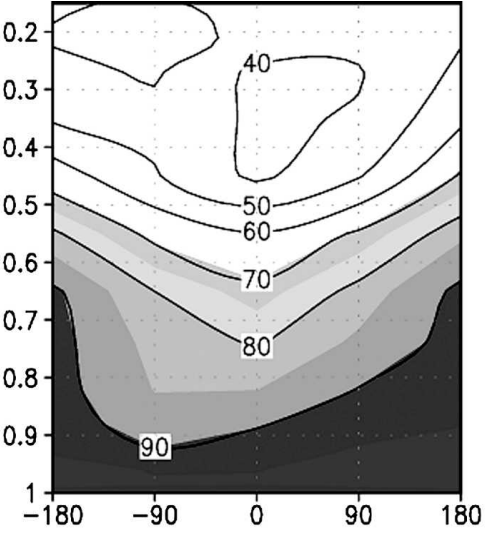

(c)

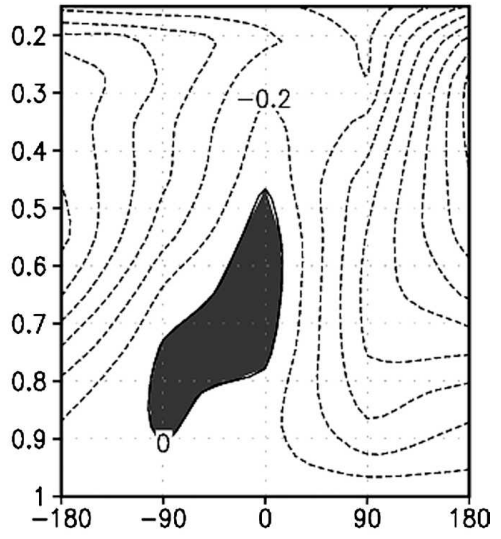

(f)

FIG. 7. Composite of five development phases in Exp3 for (a) the vorticity $\left(10^{-5} \mathrm{~s}^{-1}\right)$, (b) divergence $\left(10^{-5} \mathrm{~s}^{-1}\right)$, (c) relative humidity $(\%)$, (d) equivalent potential temperature $(\mathrm{K})$, (e) latent heat rate $\left(10^{-4} \mathrm{~K} \mathrm{~s}^{-1}\right)$, and (f) vertical velocity $\left(10^{-5} \mathrm{~s}^{-1}\right)$. The time evolution in the horizontal axis is from a convective active phase $\left(-180^{\circ}\right)$ to a break phase $\left(0^{\circ}\right)$ and then to a new active phase $\left(180^{\circ}\right)$. The vertical axis is the sigma level.

The atmospheric moisture transport regulates the strength of convection through controlling the atmospheric stratification, precipitation-induced downdraft, PBL divergence, and low-level vorticity generation.
Figure 8 is a schematic diagram, illustrating the role of the PBL moisture discharge/recharge in the oscillatory TC development. The water vapor has both positive and negative impacts on the vorticity building-up at

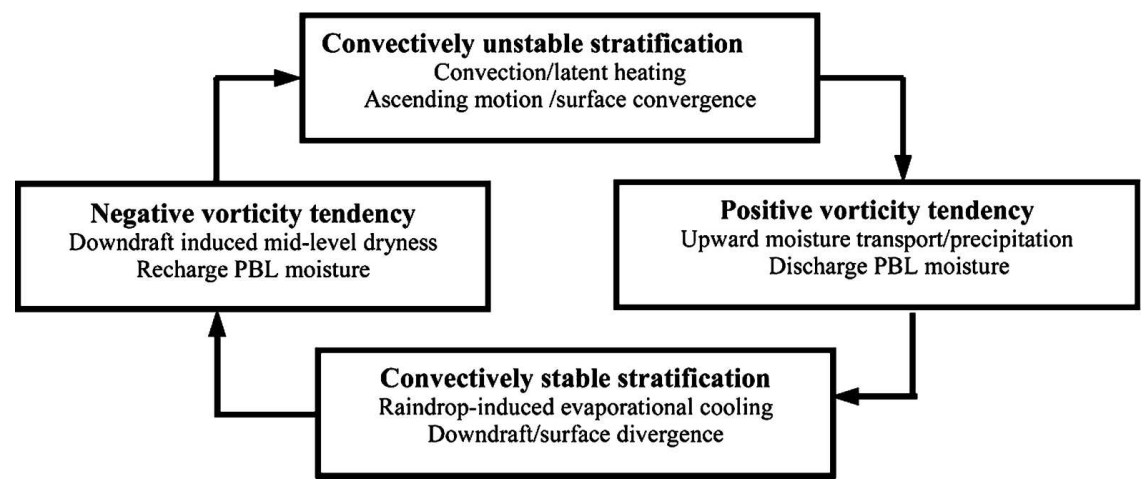

FIG. 8. Schematic diagram illustrating the role of PBL moisture recharge/discharge in the TC oscillatory development. 


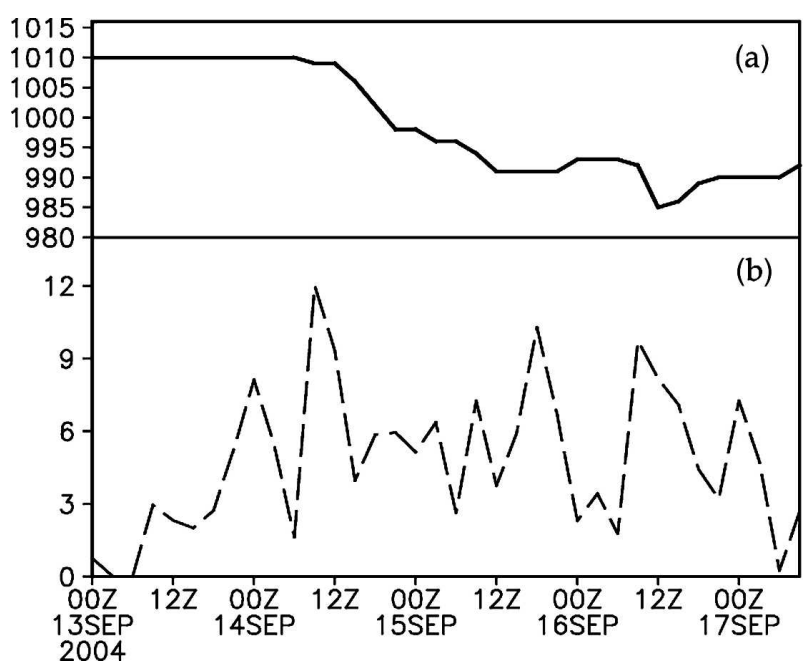

FIG. 9. Time evolution of (a) minimum sea level pressure (hPa) and (b) averaged TRMM rain rate $\left(\mathrm{mm} \mathrm{h}^{-1}\right)$ within $1^{\circ}$ radius of Hurricane Jeanne during 13-17 Sep 2004.

different phases. On one hand, strong ascending motion associated with deep convection transports moisture from PBL to upper troposphere, which leads to the discharge of PBL moisture and consequently a convectively more stable stratification. On the other hand, the convection-induced raindrops evaporate, leading to midlevel cooling and downdraft. The down- draft further leads to dryness (through vertical advection) and thus a reduction of equivalent potential temperature in the middle-to-lower troposphere. This reduction along with the recharge of PBL moisture due to surface evaporation leads to the reestablishment of a convectively unstable stratification and thus new convection.

To examine whether the model oscillatory TC development feature appears in nature, we have conducted an observational analysis using 3-hourly TRMM rainrate data (at a horizontal resolution of $0.25^{\circ} \times 0.25^{\circ}$ ). We selected Hurricane Jeanne (13-17 September 2004) as an example. Jeanne was officially warned as a tropical depression at 1500 UTC on 13 September. According to Gray (1998), the magnitude of convection concentration near the core region is a crucial factor for perturbation development. This prompted us to examine the evolution of rainfall near the core region. We examined the time series of rainfall rate averaged within $1^{\circ}$ radius of the TC center, and used the averaged rainfall rate to represent the strength of convection in the TC core region. The TC center is determined based on TC best-track data available at http:// weather.unisys.com/hurricane/index.html. Before the warning, the TC center is determined based on maximum low-level vorticity derived from the QuikSCAT data. Figure 9 shows the time evolution of the averaged

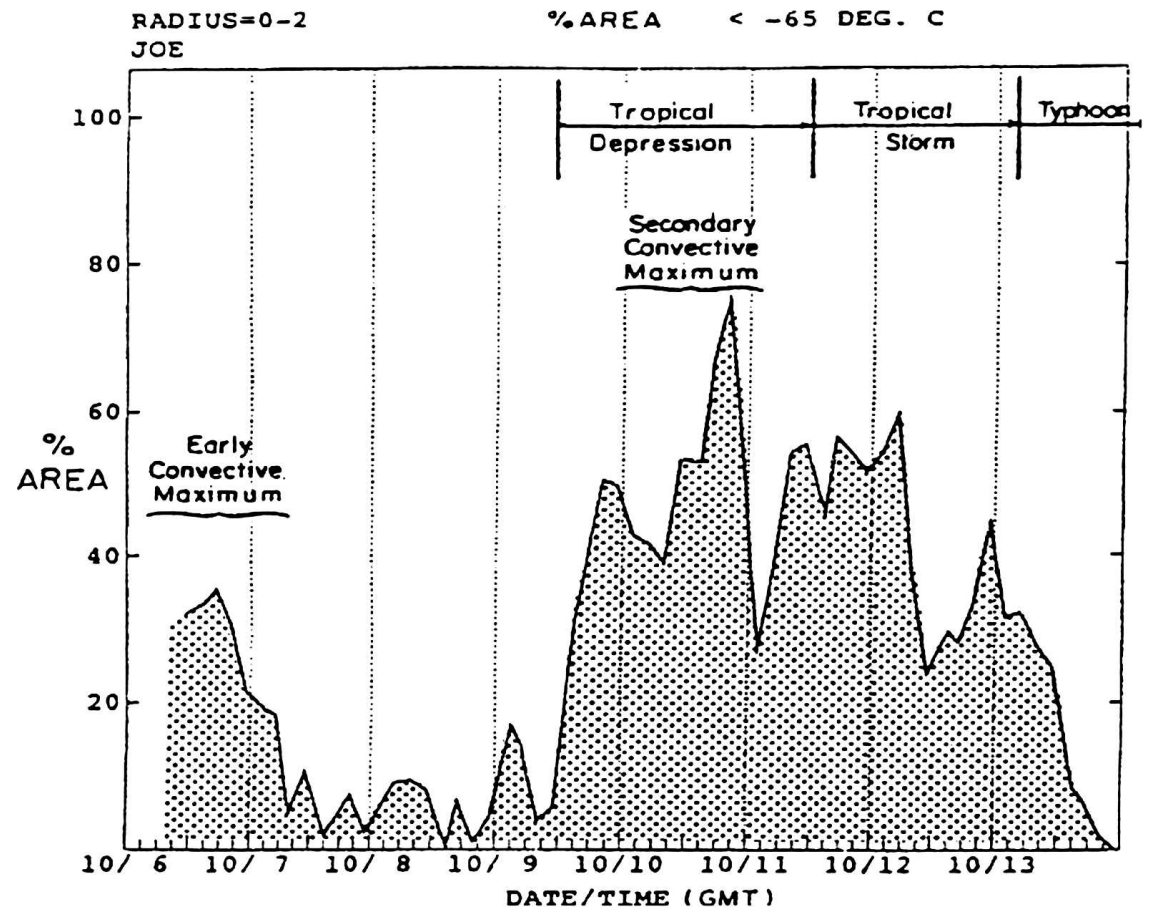

FIG. 10. Temporal change of percentage of the area of IR cloud-top temperature less than $-65^{\circ} \mathrm{C}$ within a $220-\mathrm{km}$ radius from the center of TC Joe in the western North Pacific (from Zehr 1992). 

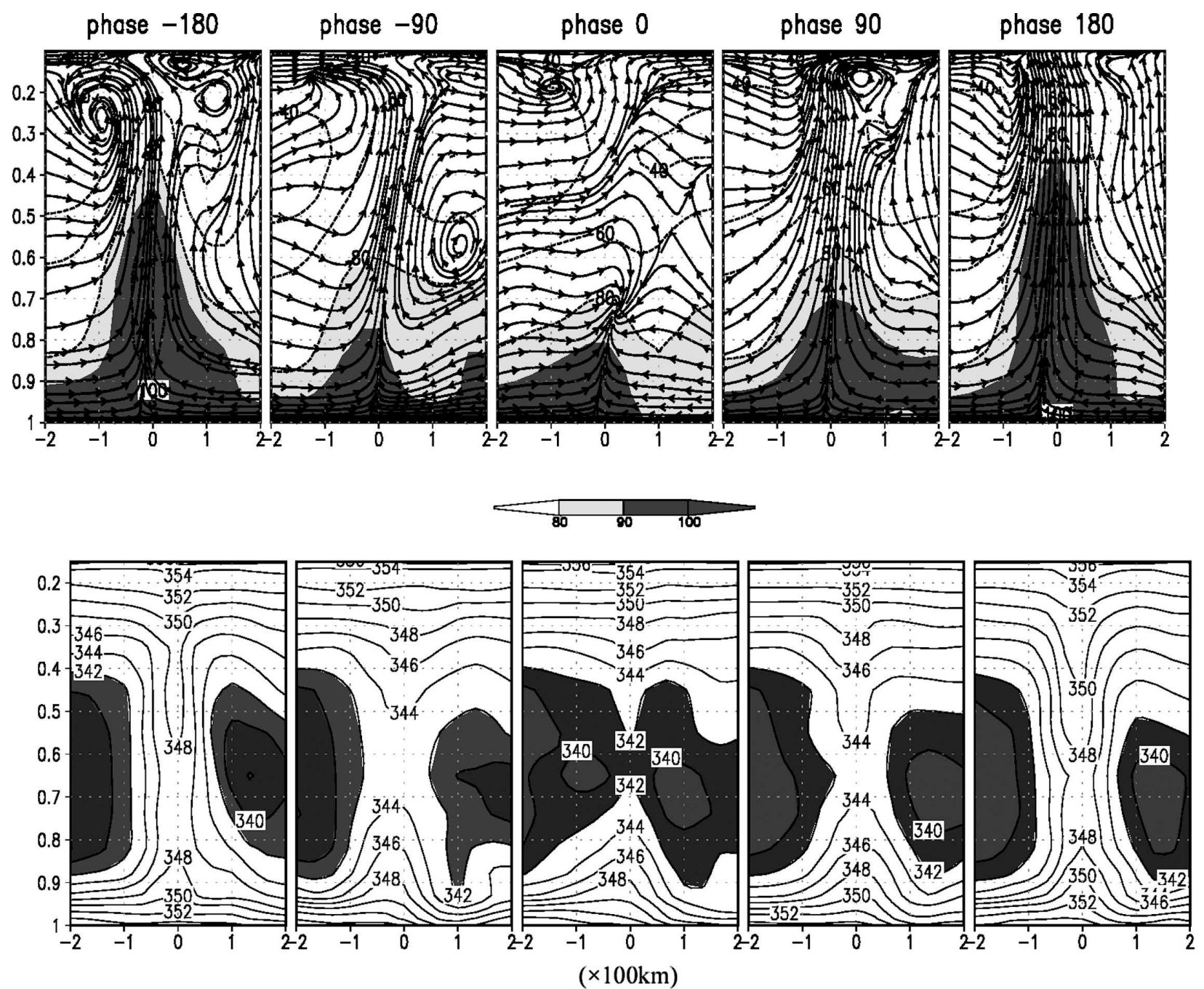

FIG. 11. (top) North-south vertical cross section of streamline (vector) and relative humidity ( $>80 \%$ shaded) and (bottom) equivalent potential temperature (contour interval is $2 \mathrm{~K}$ ) fields across the low-level vorticity center at different oscillatory development phases in Exp3.

rainfall rate (dashed line), along with the change of Jeanne's minimum sea level pressure. One can see that the averaged rain rate exhibits a clear oscillatory evolution. The oscillation period is ranged from 9 to $18 \mathrm{~h}$, consistent with the model results.

Another observational support of the oscillatory development is inferred from the evolution of cloud-top temperature for Typhoon Joe in the western North Pacific (Zehr 1992). Figure 10, originally from Zehr (1992), shows that the percentage of the area of cloudtop IR temperature lower than $-65^{\circ} \mathrm{C}$ within a $220-\mathrm{km}$ radius of the TC center exhibits an oscillation character. The period of the oscillation is around $12 \mathrm{~h}$, similar to that in the model. Thus, both the TRMM rain-rate and cloud-top temperature provide observational evidences of the TC oscillatory development in the real world, consistent with what we found in numerical model simulations.

Figure 11 shows the composite north-south vertical cross section of streamline and relative humidity fields during the convectively active and break phases. Deep convection penetrates through the troposphere at phase $-180^{\circ}$, leading to upward transport of water vapor. As a result, $80 \%$ relative humidity layer reaches up to $400 \mathrm{hPa}$. The convection starts to weaken at phase $-90^{\circ}$, as the updraft branch starts to tilt and a subsidence branch starts to develop in the middle troposphere to the north of the convection center. The effect of the downdraft can be inferred from the relative humidity field. There are significant decreases in both the amplitude and the height of the moist layer. The development of the midtropospheric downdraft eventually 




FIG. 12. East-west vertical cross section of vertical velocity $\left(10^{-5} \mathrm{~s}^{-1}\right)$ field across the low-level vorticity center at different oscillatory development phases in Exp3.

leads to the disconnection of the upper-tropospheric convective branch and PBL moisture at phase $0^{\circ}$. The raindrop-induced evaporative cooling and downdraft lead to the reduction of equivalent moist potential temperature in the lower troposphere, triggering the subsequent development of new convection.

The east-west vertical cross section shown in Fig. 12 exhibits similar structure and evolution characters. The major downdraft branch starts to develop in the east side of the convection center, moving upward from phase $-180^{\circ}$ to $-90^{\circ}$. Meanwhile, the updraft branch over the vortex center also migrates upward while weakening. At phase $0^{\circ}$ maximum upward motion is located at $250 \mathrm{hPa}$. New convection starts to develop at phase $90^{\circ}$, and reaches its maximum amplitude at phase $180^{\circ}$.

The low-level vorticity and divergence fields exhibit a rather complex phase relationship during different development stages. While the convergence center is well collocated with the cyclonic vorticity center during the convectively active phase, they have a completely opposite phase relationship at the break phase and mismatch in between (Fig. 13). The difference in the vorticity-divergence phase relationship is well reflected in the rainfall field. Although the vorticity difference between the active and break phases is relatively small, the rainfall amplitude exhibits a large difference (top panel of Fig. 13). A similar feature appears in the latent heat field (not shown).

\section{Sensitivity to initial moisture profile, model resolution, and background flow pattern}

In the previous experiments, the background relative humidity near the MG center is set to be slightly higher than the surrounding (left panel of Fig. 14). Physically, it is argued that the RH increase near the MG center is attributed to frictional moisture convergence induced by cyclonic vorticity at the top of PBL. To demonstrate the importance of initial background moisture conditions, here we conduct a sensitivity experiment (Exp4) in which the initial MG fields are kept the same as those in Exp3 except that the moisture profile is set to be flat at each level (see the right panel of Fig. 14).

The numerical experiment shows that the model cyclogenesis is very sensitive to the initial moisture condition. With the reduction of the initial background moisture near the MG center, no cyclogenesis occurs in the wave train. Figure 15 shows the evolution of the surface pressure field in this case. A minimum surface pressure of $1004 \mathrm{hPa}$ appears at day 3. After that, the low pressure system starts to weaken. The corresponding surface wind has a similar evolution feature and is generally weak.

Figure 16 shows the time-vertical section of areaaveraged vorticity, relative humidity and vertical velocity fields. Comparing Figs. 6 and 16, one may notice that the vorticity oscillating development feature appears in both the genesis and nongenesis cases. A fundamental difference lies in that in the genesis case, the vorticity penetrates to high altitude, whereas in the nongenesis case the vorticity is confined to the PBL. A similar feature is found in the relative humidity field. Compared to the genesis case in which the moisture can penetrate into much deeper layers, the moist layer in the nongenesis case is much shallower. The results above imply that the vertical transport of the low-level vorticity and moisture might be crucial for continuous TC development. 

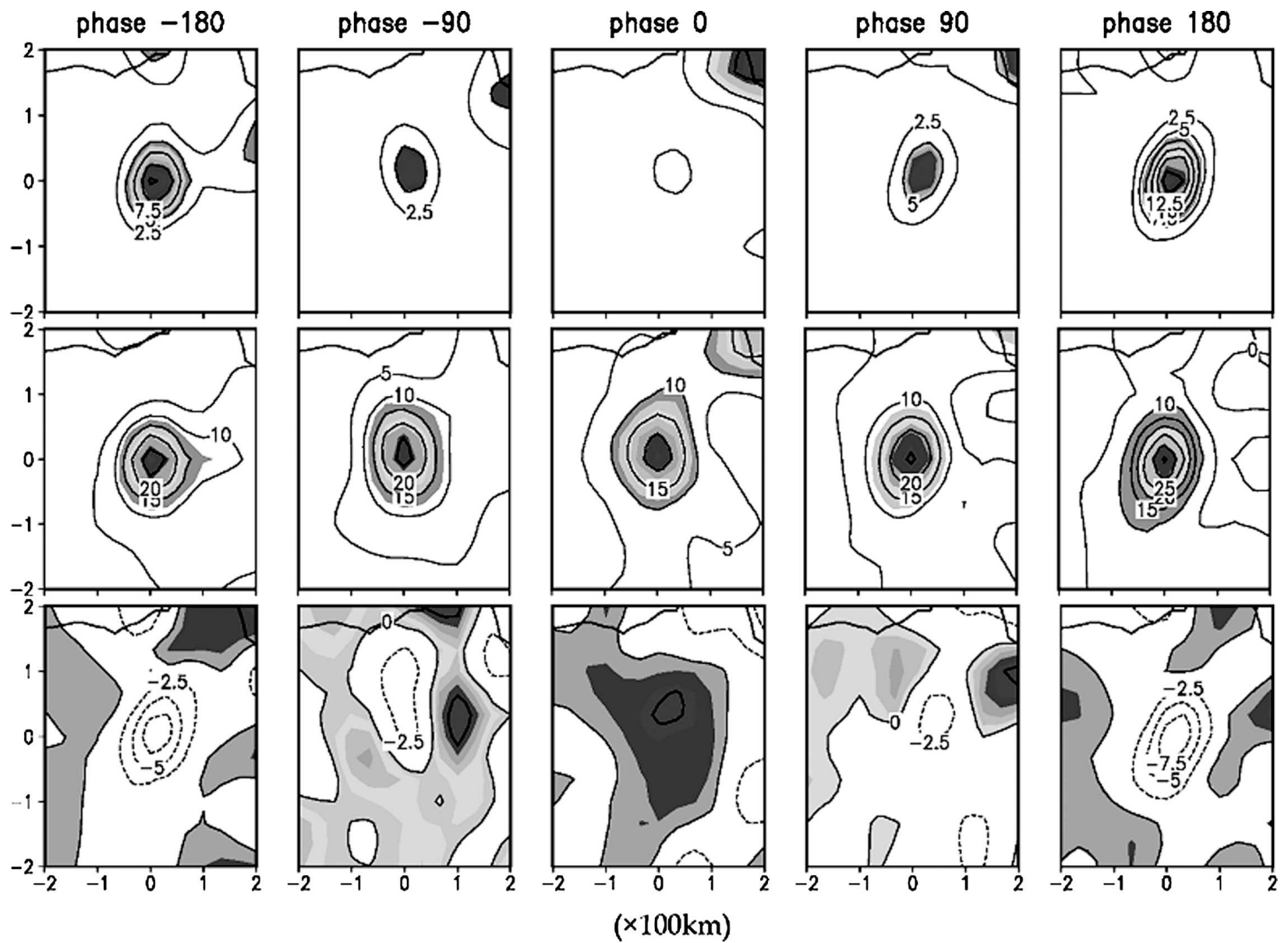

FIG. 13. Phase evolution of the composite (top) rainfall field $\left(\mathrm{mm} \mathrm{h}^{-1}\right)$, (middle) relative vorticity $\left(10^{-5} \mathrm{~s}^{-1}\right)$, and (bottom) divergence $\left(10^{-5} \mathrm{~s}^{-1}\right)$ fields at $\sigma=0.82$ from Exp3.
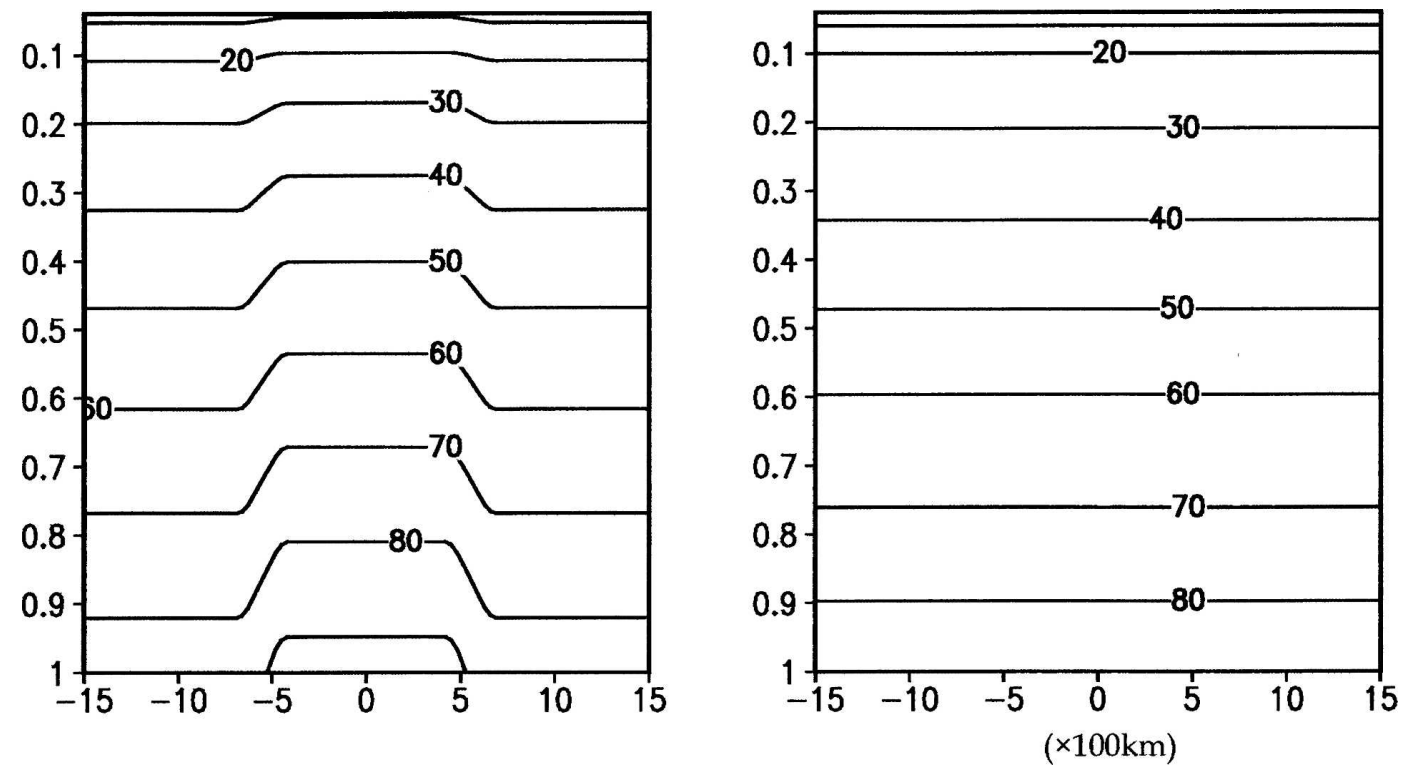

FIG. 14. Vertical cross section of initial background relative humidity (\%) fields specified in (left) Exp3 and (right) Exp4. 


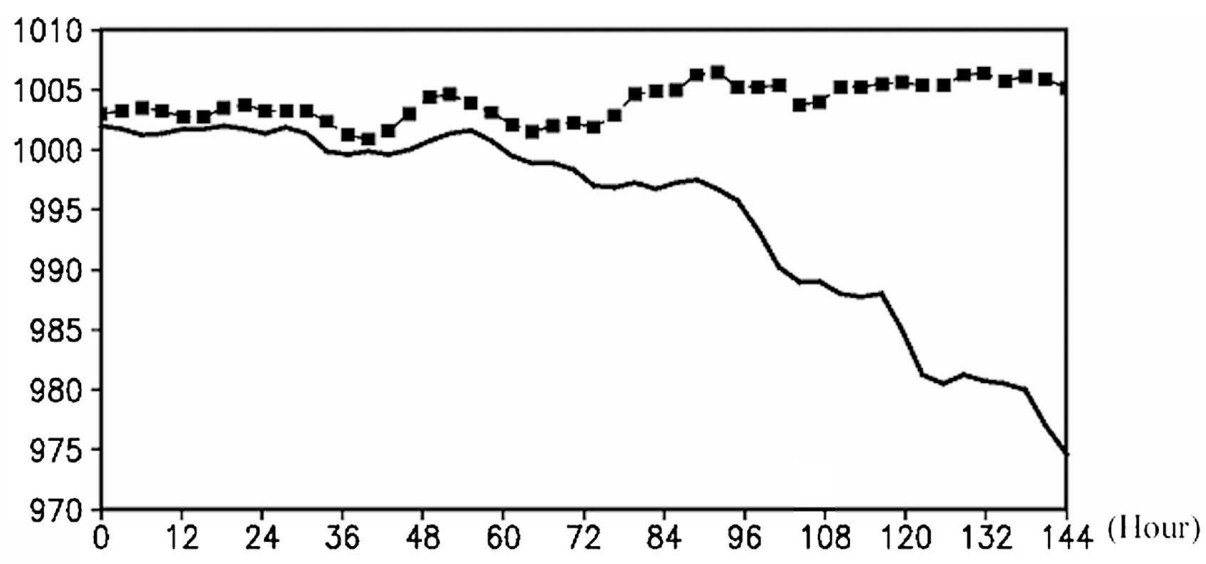

DAY 1

DAY 3

DAY 5


FIG. 15. (top) Time evolution of the minimum sea level pressure in Exp4 (dotted line) and Exp3 (solid line). (bottom) The surface pressure patterns at days 1,3, and 5 in Exp4.

How does the difference in the initial moisture profile impact the subsequent TC development? In the following we address this question from both dynamic and thermodynamic points of view. From a purely dynamic point of view, the initial moisture difference may account for the subsequent difference in the strength of the background vertical motion. To demonstrate this dynamic impact, we conduct an additional experiment (Exp5) in which the initial fields of the MG are kept same as those in Exp2 except that a flat RH distribution (as shown in the right panel of Fig. 14) is used. Figure 17 shows the averaged vertical motion and divergence fields during initial $48 \mathrm{~h}$ from Exp5 and Exp2. Indeed, the change of the initial RH field impacts the subsequent development of the background vertical motion. A larger initial moisture distribution leads to stronger background ascending motion over the monsoon gyre region. It is argued that a stronger vertical transport of perturbation vorticity and moisture by the background ascending motion may lead to the continuous growth of the perturbation to overcome natural dissipation induced by convective downdrafts.

From a thermodynamic point of view, a deeper initial moisture layer is a necessary condition to cause water vapor condensation and latent heat release, the latter of which is essential to overcome adiabatic cooling induced by ascending motion and maintain the convection. Keep in mind that the convective instability induced by downdraft-induced dryness in the middle troposphere might trigger convection, but the convection would not be maintained without release of convective heating. If middle-tropospheric RH is not sufficiently large to lead to latent heat release, given the same dynamic triggering (say, a cyclonic vorticity perturbation at the top of PBL), Ekman-pumping-induced ascending motion could damp the initial vorticity, preventing the further development of the perturbation. Compared with the flat $\mathrm{RH}$ case in Exp4, the monsoon gyre with a higher RH (Exp3) would lead to a decrease of the radius of Rossby deformation due to the reduction of 

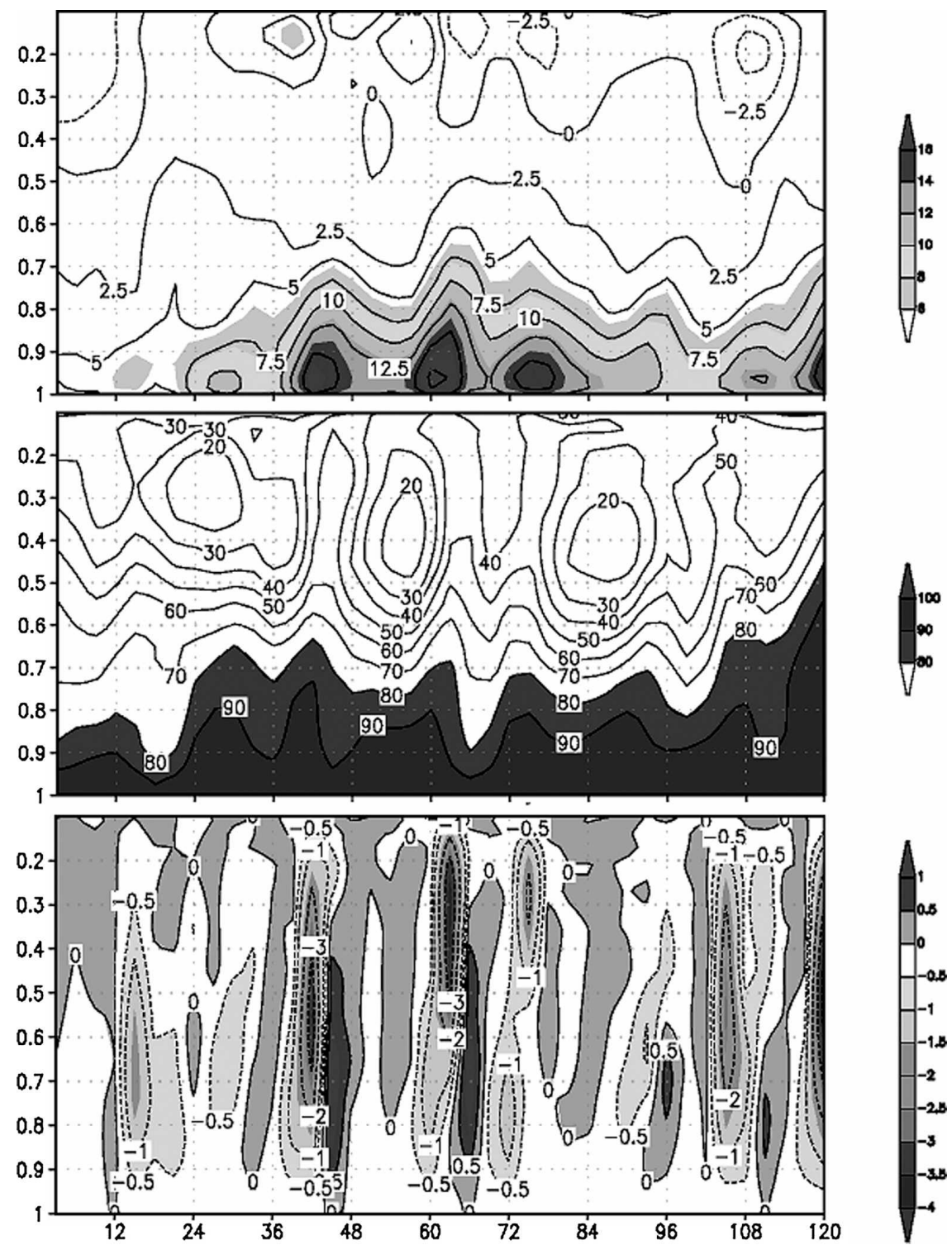

FIG. 16. Time-vertical section of area-averaged (top) relative vorticity $\left(10^{-5} \mathrm{~s}^{-1}\right)$, (middle) relative humidity (\%), and (bottom) vertical motion $\left(10^{-5} \mathrm{~s}^{-1}\right)$ in Exp4. The average is calculated in a $120 \mathrm{~km} \times 120 \mathrm{~km}$ domain centered at the low-level maximum vorticity. The horizontal axis is time in hours and the vertical axis is the sigma level.

the moist static stability. As a result, the vortex becomes more efficient in generating rotational momentum in response to the local warming (Hack and Schubert 1986).

The consequence of the initial moisture difference may be further inferred from the evolution of the vertical velocity and relative humidity fields in the genesis and nongenesis cases (Fig. 18). They oscillate around a constant reference level in the nongenesis case (Exp4), but experience a rapid growth after initial oscillations in Exp3. This suggests that TC genesis may exhibit two development stages. The first is an oscillatory development stage-most of the tropical disturbances may experience this stage owing to the moisture discharge/ recharge and the convection-circulation feedback. Whether the perturbation can continue to grow depends on whether it can reach the second stage, in which a preferred background may help push it away from the original reference level, leading to a rapid intensification toward a new equilibrium state.

The result from Exp1 (with a uniform 15-km grid) shows that the TCED-induced Rossby wave train can- 

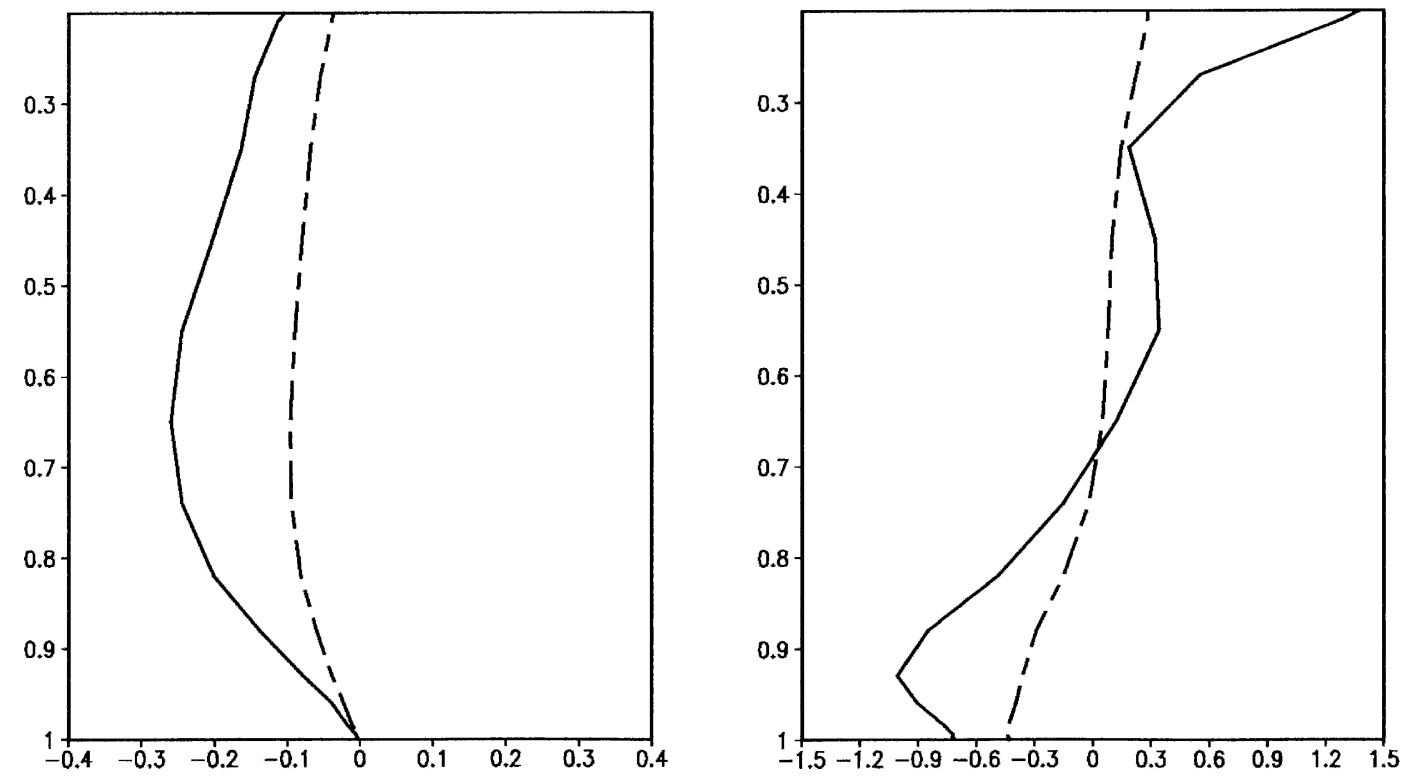

FIG. 17. Vertical profiles of (left) background vertical motion $\left(10^{-5} \mathrm{~s}^{-1}\right)$ and (right) divergence fields $\left(10^{-5} \mathrm{~s}^{-1}\right)$ averaged during initial $48 \mathrm{~h}$ over the monsoon gyre center (within a 300-km radius) in Exp2 (solid line) and Exp5 (dashed line). The vertical axis is the sigma level.

not lead to cyclogenesis in a resting environment. To examine whether this conclusion is resolution dependent, we performed an additional experiment (Exp6) in which the model has two nested meshes at the resolution of 15 and $5 \mathrm{~km}$, respectively, with the finer mesh covering the potential cyclogenesis region (i.e., over the cyclonic vorticity region of the wave train). Figure 19 shows the evolution of the surface pressure field simulated by this nested model. After an initial deepening of the low pressure system in the wave train during the first four days, it slowly decays. At day 7 , there are no clear closed circulation and a low-pressure center in the wave train. The sensitivity experiment supports the conclusion above, that is, the Rossby wave train alone cannot lead to cyclogenesis, and a proper background flow/moisture condition is necessary for the cyclogenesis induced by the TCED.

In Exp3 an idealized MG background flow is specified. To examine the sensitivity of the cyclogenesis to the mean flow, we conducted an experiment (Exp7) in which a background monsoon shear line flow pattern (Fig. 20a) is specified. Same as in Exp3, the relative humidity is slightly increased over the cyclonic vorticity center (see the shading region for the $\mathrm{RH}$ difference). This idealized monsoon shear line pattern is similar to the observed composite in the western North Pacific (Ritchie and Holland 1999; Fig. 20b). Initially, the same Rossby wave train perturbation is specified. The numerical result shows that the Rossby wave trainmonsoon shear line interaction can also lead to the gen- esis of a new TC. At day 5, the surface minimum central pressure reaches below $980 \mathrm{hPa}$ (Fig. 20c). However, with a flat background $\mathrm{RH}$ distribution as in Fig. 14b, no cyclogenesis occurs in the model, which again points out the importance of the proper initial moisture condition in tropical cyclogenesis.

\section{Conclusions and discussion}

The cyclogenesis events associated with the Rossby wave energy dispersion of a preexisting TC are simulated in a $3 \mathrm{D}$ model. Our numerical results point out the importance of the mean monsoon flow (such as the monsoon gyre and the monsoon shear line patterns) in leading to TC development in the Rossby wave train. The simulated new TC has realistic dynamic and thermodynamic structures.

Of particular interest is the evolution of low-level vorticity associated with the cyclogenesis. It is characterized by oscillatory growth. The discharge and recharge of the atmospheric PBL moisture and its interaction with convection and circulation are responsible for this oscillatory vorticity development. Our composite analysis suggests that water vapor transport regulates the strength of convection through controlling the atmospheric convective instability and stratification, raindrop-induced evaporative cooling and downdraft, PBL divergence, and low-level vorticity generation. It has both positive and negative impacts on the vorticity tendency at different phases. On one hand, 



FIG. 18. Time evolution of vertical velocity (solid line, $10^{-5} \mathrm{~s}^{-1}$ ) and relative humidity (dashed line, \%) averaged in middle troposphere at (top) a cyclogenesis case (Exp3) and (bottom) a nongenesis case (Exp4).

strong ascending motion associated with deep convection transports moisture from PBL to upper troposphere, leading to the discharge of PBL moisture and a convectively stable stratification. On the other hand, the convection-induced raindrops evaporate, leading to midlevel cooling and downdraft. The downdraft further leads to dryness (through vertical advection) and thus a reduction of equivalent potential temperature. This reduction along with the recharge of PBL moisture due to surface evaporation leads to reestablishment of a convectively unstable stratification and thus new convection.

The oscillatory development character revealed from the numerical simulations is confirmed by the time series of TRMM rainfall rate and cloud-top IR temperature averaged near the core region of Hurricane Jeanne (2004) and Typhoon Joe. The discovery of this oscillatory development is important for understanding cyclogenesis because it may help explain why $90 \%$ of tropical disturbances do not grow into a TC. A traditional view of TC development is the positive feedback between the primary and secondary circulation in presence of convective heating. This positive feedback would lead to exponential growth of the tropical perturbation. However, our model simulations show that during the initial development stage, the vorticity exhibits an oscillatory evolution. This oscillatory development is closely associated with the interplay between convective updraft and raindrop-evaporation-induced downdraft and discharge and recharge of moisture in the PBL. Many tropical disturbances dissipate after initial development, either because of interferences from other scale motions or simply because of unfavorable environmental conditions that are unable to sustain new convection after precipitation-induced downdraft dries up the middle troposphere (Bister and Emanuel 1997; Bister 2001). Only when reaching a certain critical level, the perturbation can grow exponentially into a
Day 1

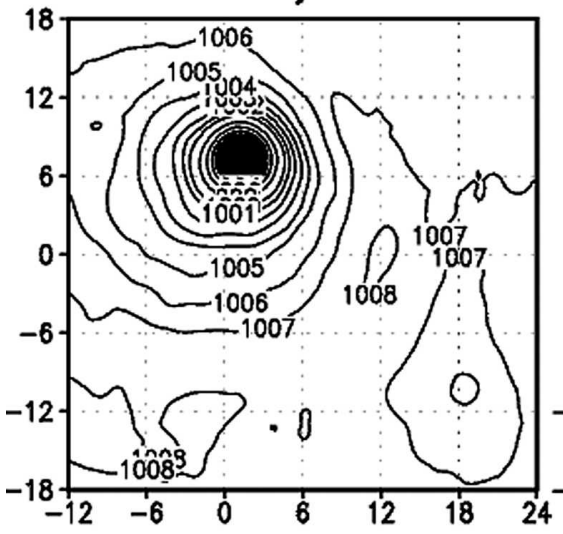

Day 4

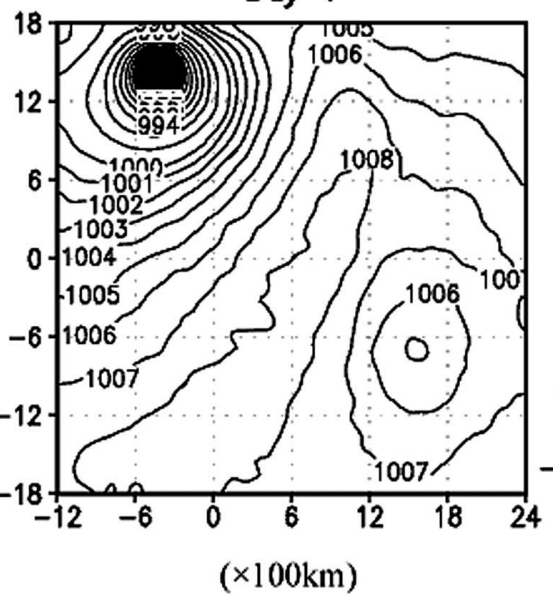

Day 7

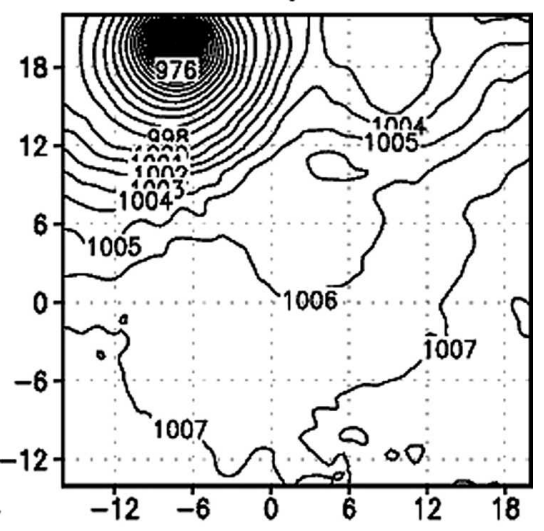

FIG. 19. Simulated surface pressure (hPa) patterns at days 1 , 4, and 7 from Exp6. Horizontal distance has a unit of 100 km, and $(0,0)$ denotes the center of the model domain. 

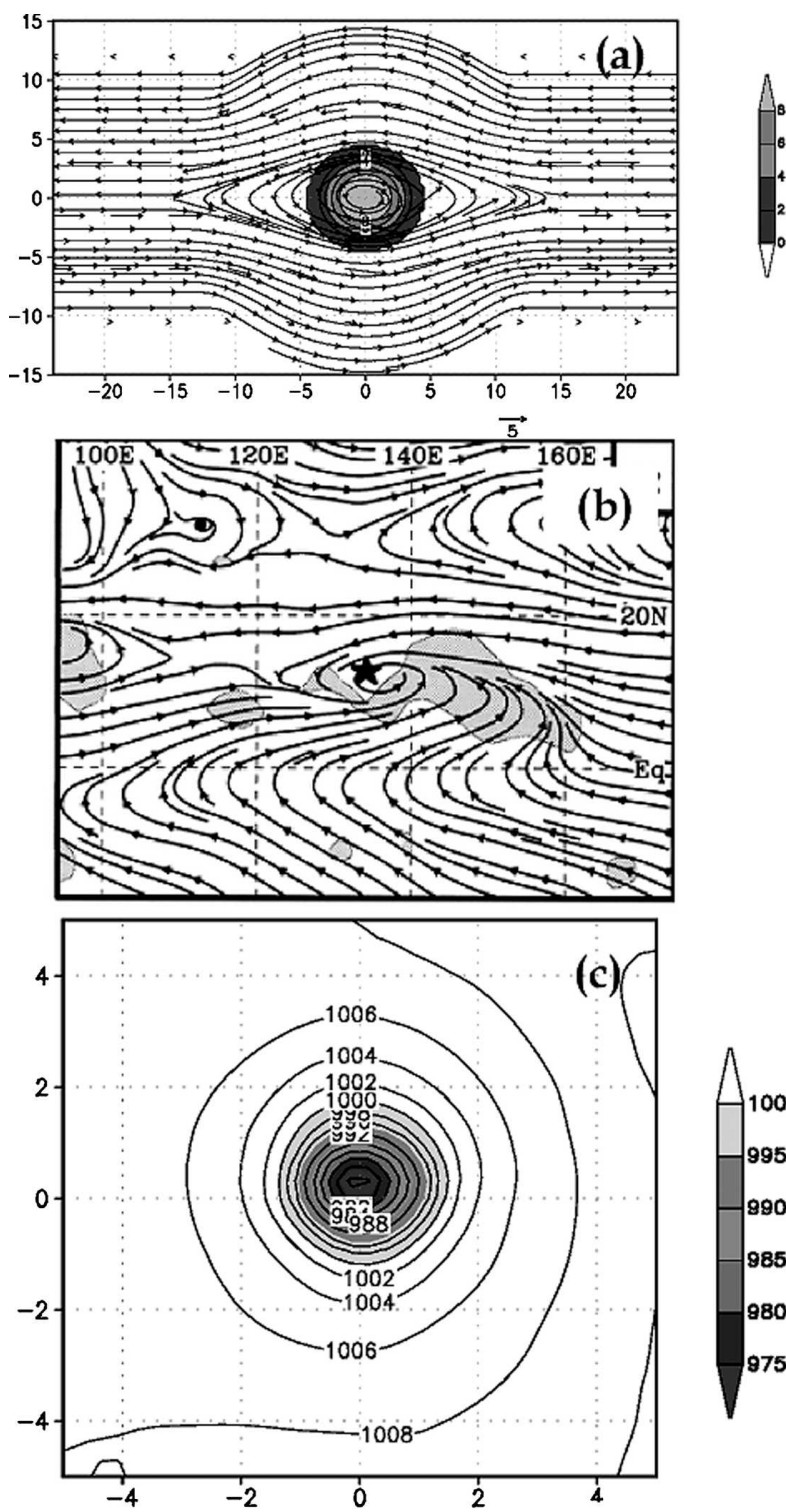

FIG. 20. (a) Idealized monsoon shear line pattern (the surface relative humidity difference from the environment is shaded with units of \%); (b) observed monsoon shear line pattern from Ritchie and Holland (1999); and (c) surface pressure (hPa) field at day 5 in Exp7. Horizontal distance has a unit of $100 \mathrm{~km}$ relative to the monsoon shear line center for (a) and simulated TC center for (c).

new equilibrium state. In reality many perturbations may oscillate for a while and then die out, because of unfavorable environmental conditions.

An open question is what sets up the oscillation period. The averaged period from the model simulations is around $12 \mathrm{~h}$, which is close to an oscillatory period derived from a T1279L96 (10-km horizontal resolution) global model (run at the Earth Simulator, see Yoshioka et al. 2004). Given that the two models contain completely different physical packages (e.g., the global model contains the diurnal cycle of the solar radiation while the current model does not) and initial conditions, it is conceivable that the oscillatory period is set up by fundamental TC dynamics and the nature of the moisture-convection-circulation feedback. To ensure that the oscillatory period is not affected by reflection of waves in the lateral boundaries, sensitivity experiments with a larger domain $(6000 \mathrm{~km}$ by $6000 \mathrm{~km})$ are carried out, and results show that the period is not sensitive to the change of the domain size.

The comparison of the first two experiments (Exp1 and Exp2) and the third experiment (Exp3) implies the importance of the WISHE mechanism in TC genesis, this is, a sufficiently large wind speed and thus sufficiently large surface heat fluxes are important for vortex development. The contrast between Exp3 and Exp4 shows that even given the same surface wind speed, a reduced background moisture distribution may lead to no cyclogenesis. This points out that the initial moisture content is another crucial factor that regulates TC genesis. These results are consistent with previous studies by Stein and Alpert (1993) and Emanuel (1994), who pointed out that a necessary condition for TC genesis is the establishment of an order 100-km-wide column of nearly saturated air in the core of the system. They are also consistent with our observational analysis in Part I that the midtropospheric background moisture is an important regulating factor for TCED-induced cyclogenesis. Therefore, it is necessary to take into account both the surface wind speed and moisture effects.

To further illustrate the importance of the background moisture effect, we conducted an additional experiment (Exp8) in which the MG moisture field is present but there is no MG flow. Figure 21 shows the surface pressure evolution at this case. A TC-like vortex is generated, even though it takes longer time ( 9 days) to reach $989 \mathrm{hPa}$ compared to only 4-5 days in Exp3. The combined numerical results above suggest that a criterion for cyclogenesis depends on both the surface wind speed and environmental moisture conditions.

The discharge-recharge paradigm differs from the original WISHE theory in that the latter assumes a neutral stratification, whereas the current study emphasizes the time evolution of middle tropospheric temperature and moisture and thus the temporal change of atmospheric stratification at various development phases. The change of the stratification is closely related to the discharge and recharge of atmospheric moisture in PBL. It is found that relative humidity during the suppressed convective (or downdraft) phase is reduced rather than increased. This is surprising because raindrop-induced evaporation may lead to the cooling and 

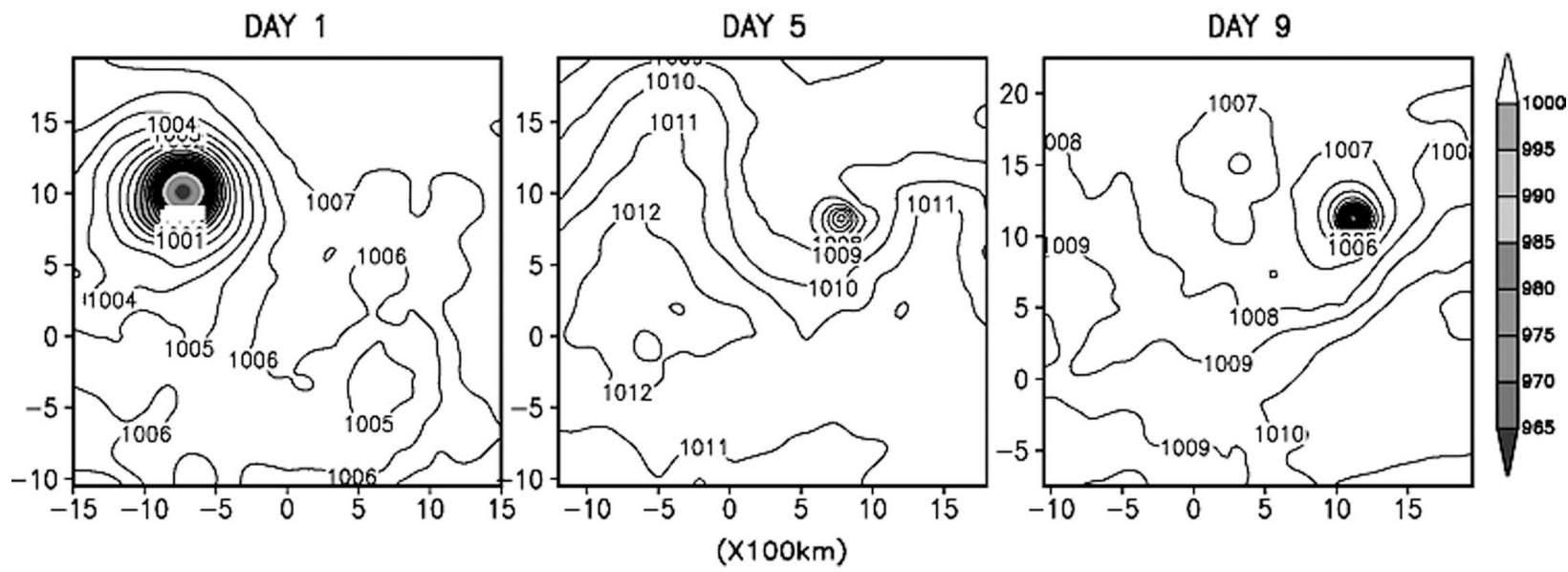

FIG. 21. Surface pressure (hPa) patterns at days 1, 5, and 9 from Exp8. Horizontal distance has a unit of $100 \mathrm{~km}$, with $(0,0)$ denoting the center of the model domain.

wetness and thus the increase of middle-level relative humidity. The decrease of relative humidity in the model is primarily attributed to the vertical advection of dry air by downdraft. As a result of the dryness and cooling, saturation equivalent potential temperature at midlevel decreases, resulting an increase in the convective instability. The decrease of relative humidity during the convectively break phase implies that the dryness induced by vertical advection exceeds the effect of raindrop-induced evaporation.

Our numerical simulations reveal a time-dependent vorticity-divergence relationship during the TC development stage, which differs from a traditional view that during the TC development low-level vorticity collocates with the PBL convergence because of the Ekman pumping effect so that there is a positive feedback between the vorticity and convergence. We note that the in-phase temporal relationship between the vorticity and convergence appears only in the convectively active phase, but not in the opposite phase. The change of this phase relationship is closely related to the oscillatory development of a TC.

Our numerical experiments suggest that the TCEDinduced cyclogenesis can occur only when a preferred mean flow is presented. The wave train itself cannot lead to TC genesis in a resting environment. This is consistent with the observational analysis in Part I, but different from the result of Holland (1995). The difference lies in the convective heating schemes. In Holland (1995) the heating is simply proportional to low-level vorticity, whereas in the current model, a combination of an explicit heating scheme and a mass flux scheme is used.

Another issue is how to quantitatively measure the effect of the background flow in the cyclogenesis. In the current setting, the synoptic wave train and the background mean flow are introduced initially and there are full interactions between the synoptic perturbation and the mean flow. As the mean flow also involves time, it is difficult to separate the two components during the model integration. It might be useful to develop an anomaly model (e.g., Li et al. 2003) in which a timeindependent $3 \mathrm{D}$ basic state is specified so that one can readily diagnose the mean flow effect on the perturbation growth.

The oscillatory development in the model motivates us to examine structure and evolution characteristics of satellite-measured 3D water vapor fields prior to TC formation. We are currently analyzing the phase relationship among the atmospheric moisture, vorticity, divergence, vertical motion, and stratification using the newly available Aqua data, and simulating real-case cyclogenesis events associate with the TCED using the fifth-generation Pennsylvania State UniversityNational Center for Atmospheric Research Mesoscale Model (MM5). These results will be reported elsewhere.

Acknowledgments. The authors thank Drs. Melinda Peng and Yuqing Wang for valuable discussions and anonymous reviewers for constructive comments. This work was supported by NSF Grant ATM01-19490 and ONR Grants N000140310739 and N000140210532. International Pacific Research Center is partially sponsored by the Japan Agency for Marine-Earth Science and Technology (JAMSTEC).

\section{REFERENCES}

Bister, M., 2001: Effect of peripheral convection on tropical cyclone formation. J. Atmos. Sci., 58, 3463-3476. 
—_, and K. A. Emanuel, 1997: The genesis of Hurricane Guillermo: TEXMEX analyses and a modeling study. Mon. Wea. Rev., 125, 2662-2682.

Carr, L. E., and R. L. Elsberry, 1995: Monsoonal interactions leading to sudden tropical cyclone track changes. Mon. Wea. Rev., 123, 265-289.

Chan, J. C.-L., and R. T. Williams, 1987: Analytical and numerical studies of the beta-effect in tropical cyclone motion. Part I: Zero mean flow. J. Atmos. Sci., 44, 1257-1265.

Detering, H. W., and D. Etling, 1985: Application of the E- $\varepsilon$ turbulence model to the atmospheric boundary layer. Bound.Layer Meteor., 33, 113-133.

Emanuel, K. A., 1989: The finite-amplitude nature of tropical cyclogenesis. J. Atmos. Sci., 46, 3431-3456.

_ 1994: The physics of tropical cyclogenesis over the eastern Pacific. Tropical Cyclone Disasters, J. Lighthill, Z. Zhemin, G. Holland, and K. Emanuel, Eds., Peking University Press, 588 pp.

Fairall, C. W., E. F. Bradley, D. P. Rogers, J. B. Edson, and G. S. Young, 1996: Bulk parameterization of air-sea fluxes in TOGA COARE. J. Geophys. Res., 101, 3747-3767.

Fiorino, M., and R. L. Elsberry, 1989: Contributions to tropical cyclone motion by small, medium, and large scales in the initial vortex. Mon. Wea. Rev., 117, 721-727.

Flierl, G. R., 1984: Rossby wave radiation from a strongly nonlinear warm eddy. J. Phys. Oceanogr., 14, 47-58.

Gray, W. M., 1998: The formation of tropical cyclones. Meteor. Atmos. Phys., 67, 37-69.

Hack, J. J., and W. H. Schubert, 1986: Nonlinear response of atmospheric vortices to heating by organized cumulus convection. J. Atmos. Sci., 43, 1559-1573.

Hendricks, E. A., M. T. Montgomery, and C. A. Davis, 2004: On the role of "vortical" hot towers in the formation of tropical cyclone Diana (1984). J. Atmos. Sci., 61, 1209-1232.

Holland, G. J., 1995: Scale interaction in the western Pacific monsoon. Meteor. Atmos. Phys., 56, 57-79.

Langland, R. H., and C. S. Liou, 1996: Implementation of an E- $\varepsilon$ parameterization of vertical subgrid-scale mixing in a regional model. Mon. Wea. Rev., 124, 905-918.

Li, T., and B. Fu, 2006: Tropical cyclogenesis associated with Rossby wave energy dispersion of a preexisting typhoon. Part I: Satellite data analyses. J. Atmos. Sci., 63, 1377-1389.

- — - X. Ge, B. Wang, and M. Peng, 2003: Satellite data analysis and numerical simulation of tropical cyclone formation. Geophys. Res. Lett., 30, 2122-2126.
Luo, Z., 1994: Effect of energy dispersion on the structure and motion of tropical cyclone. Acta. Meteor. Sin., 8, 51-59.

Möller, J. D., and M. T. Montgomery, 1999: Vortex Rossby waves and hurricane intensification in a barotropic model. J. Atmos. Sci., 56, 1674-1687.

—, and —, 2000: Tropical cyclone evolution via potential vorticity anomalies in a three-dimensional balance model. $J$. Atmos. Sci., 57, 3366-3387.

Reisner, J., R. M. Rasmussen, and R. T. Bruintjes, 1998: Explicit forecasting of supercooled liquid water in winter storms using the MM5 mesoscale model. Quart. J. Roy. Meteor. Soc., 124, 1071-1107.

Ritchie, E. A., and G. J. Holland, 1999: Large-scale patterns associated with tropical cyclogenesis in the western Pacific. Mon. Wea. Rev., 127, 2027-2043.

Rutledge, S. A., and P. V. Hobbs, 1984: The mesoscale and microscale structure and organization of clouds and precipitation in midlatitude cyclones. Part XII: A diagnostic modeling study of precipitation development in narrow cold-frontal rainbands. J. Atmos. Sci., 41, 2949-2972.

Shapiro, L. J., and K. V. Ooyama, 1990: Barotropic vortex evolution on a beta plane. J. Atmos. Sci., 47, 170-187.

Simpson, J., E. Ritchie, G. J. Holland, J. Halverson, and S. Stewart, 1997: Mesoscale interactions in tropical cyclone genesis. Mon. Wea. Rev., 125, 2643-2661.

Stein, U., and P. Alpert, 1993: Factor separation in numerical simulations. J. Atmos. Sci., 50, 2107-2115.

Tiedtke, M., 1989: A comprehensive mass flux scheme for cumulus parameterization in large-scale models. Mon. Wea. Rev., 117, 1779-1800.

Wang, Y., 1999: A triply nested movable mesh tropical cyclone model with explicit cloud microphysics-TCM3. BMRC Rep. 74, Bureau of Meteorology Research Center, Australia, 81 pp.

- 2001: An explicit simulation of tropical cyclones with a triply nested movable mesh primitive equation model: TCM3. Part I: Model description and control experiment. Mon. Wea. Rev., 129, 1370-1394.

_ 2002: Vortex Rossby waves in a numerically simulated tropical cyclone. Part I: Overall structure, potential vorticity, and kinetic energy budgets. J. Atmos. Sci., 59, 1213-1238.

Yoshioka, M. K., Y. Kurihara, and W. Ohfuchi, 2004: Conditions of the atmosphere and ocean for tropical cyclogenesis in its early stage. Eos, Trans. Amer. Geophys. Union, 85 (Western Pacific Geophysical Meeting Suppl.).

Zehr, R., 1992: Tropical cyclogenesis in the western North Pacific. NOAA Tech. Rep. NESDIS 16, 181 pp. 